\title{
Linear Theory of Temperature Anisotropy Instabilities in Magnetized Thermal Pair Plasmas
}

\author{
Reinhard Schlickeiser ${ }^{*}$ \\ Institut für Theoretische Physik, Lehrstuhl IV: Weltraum- und Astrophysik, Ruhr-Universität Bochum, D-44780 Bochum, \\ Germany
}

\begin{abstract}
A rigorous study of the dispersion relations of weakly amplified and weakly propagating transverse fluctuations with wave vectors $(\vec{k} \| \vec{B})$ parallel to the uniform background magnetic field $\vec{B}$ in an anisotropic bi-Maxwellian magnetized pair plasma is presented. Earlier work, based on the weakly-amplified limit, is extended to the case of weakly propagating solutions, which includes in particular aperiodic fluctuations, by the appropriate Taylor expansion of the plasma dispersion function. It is shown that six different transverse right-handed (RH) and left-handed (LH) polarized modes can be excited whose dispersion relations and instability threshold conditions are derived. The existence and instability conditions are derived in terms of the combined temperature anisotropy $A=T_{\perp} / T_{\|}$, the parallel plasma beta $\beta_{\|}=8 \pi n_{e} k_{B} T_{\|} / B^{2}$ and the electron plasma frequency phase speed $w=\omega_{p, e} /(k c)$. The pair Alfven and cyclotron instabilities are the only two weakly amplified solutions, whereas the four weakly propagating solutions are the mirror, firehose, cool magnetized Weibel and cyctronic fluctuations, respectively. The four weakly propagating solutions are aperiodic with vanishing real phase speeds $R=0$ if the plasma positrons and electrons have the same temperature anisotropy. The mirror and cool magnetized Weibel fluctuations reduce to the known hot and cool Weibel instabilities in the limit of an unmagnetized plasma.
\end{abstract}

PACS Numbers: 52.25.Xz --- 52.27.Ep --- 52.35.-g --- 94.30.cq

Keywords: Magnetized plasmas, electron-positron plasmas, waves, oscillations and instabilities.

\section{INTRODUCTION}

The explanation of the thermalization of cosmic collision-free plasmas and their near energy density equipartition with electromagnetic plasma turbulence are two challenging fundamental problems of plasma astrophysics. Thermalization by elastic two-body Coulomb collisions is orders of magnitudes too slow as compared to interactions with electric and magnetic fields because of the low-density of cosmic plasmas. The plasma parameter $g=v_{e e} / \omega_{p, e}$ is the ratio of the electron-electron Coulomb collision frequency $v_{e e}$ to the electron plasma frequency $\omega_{p, e}$, which characterizes interactions with the electromagnetic turbulence. In all cosmic plasmas, including the solar wind, the interstellar and intergalactic medium and clusters of galaxies, the plasma parameter is smaller than $10^{-10}$ (see e.g. table 8.1 in [1]), indicating that elastic collisions are not effective in establishing a local thermodynamic equilibrium. These cosmic plasmas can be regarded as effectively collision-free on the shortest time and length scales. Alternative thermalization mechanisms have to be examined such as energy diffusion by second-order Fermi interactions of charged particles with electromagnetic turbulence which are an intrinsic property of any sufficiently agitated

*Address correspondence to this author at Institut für Theoretische Physik, Lehrstuhl IV: Weltraum- und Astrophysik, Ruhr-Universität Bochum, D44780 Bochum, Germany; Tel: +49-234-3222032; Fax: +49-234-3214177; E-mail: rsch@tp4.rub.de magnetized plasma $[2,3]$. Observations in the solar wind plasma and in our own Galaxy support this hypothesis because they have established, that the energy densities of the solar wind plasma and the interstellar plasma are of the same order as the energy density of the ambient magnetic fields, cosmic rays and photon fields, and that the magnetic fields contain a dominating turbulent fraction. A full kinetic theory of the mutual particle-field interactions is required [4]. Equipartition conditions for the magnetic field energy density and the kinetic energy density of plasma particles in astrophysical sources are also often invoked for convenience [5] in order to analyze cosmic synchrotron intensities. Observationally, for a variety of nonthermal sources the equipartition concept is supported by magnetic field estimates as e.g. in the Coma cluster of galaxies [6] and radio-quiet active galactic nuclei [7].

From a theoretical point of view, there is no simple explanation of such partition. An upper limit on the magnetic field strength can be derived by applying Chandrasekhar's [8] general result, derived from the virial theorem, that for the existence of a stable equilibrium in the radiating source it is necessary that the total magnetic field energy of the system does not exceed the system's gravitational potential energy. Such a magnetic field upper limit corresponds to lower limits on the system's parallel and perpendicular plasma betas, $\beta_{\|}=8 \pi n k_{B} T_{\|} / B^{2}$ and $\beta_{\perp}=8 \pi n k_{B} T_{\perp} / B^{2}$, respectively, as bi-Maxwellian plasma distributions with different temperatures along and perpendicular to the 
magnetic field are the most likely distributions of cosmic plasmas, as observations of the solar wind plasma indicate. The solar wind plasma is the only cosmic plasma where detailed in-situ satellite observations of plasma properties are available [9]. Ten years of Wind/SWE data [10] have demonstrated that the proton and electron temperature anisotropies $A=T_{\perp} / T_{\|}$are bounded by ion cyclotron, mirror and firehose instabilities [11] at large values of the parallel plasma beta $\beta_{\|}=8 \pi n k_{B} T_{\|} / B^{2} \geq 1$. In the parameter plane defined by the temperature anisotropy $A=T_{\perp} / T_{\|}$and the parallel plasma beta $\beta_{\|}$, stable plasma configuration are only possible within a rhomb-like configuration around $\beta_{\|} \simeq 1$, whose limits are defined by the threshold conditions for the ion cyclotron, mirror and firehose instabilities. If a plasma would start with parameter values outside this rhomb-like configuration, it immediately would generate fluctuations via the ion cyclotron, mirror and firehose instabilities, which quickly relax the plasma distribution into the stable regime within the rhomb-configuration.

In order to understand the confinement limits also at small values of the parallel plasma beta $\beta_{\|}<1$ we analyze here rigorously the full linear dispersion relation in a collisionless homogenous plasma with anisotropic $(A \neq 1)$ bi-Maxwellian particle velocity distributions for electromagnetic fluctuations with wave vectors $(\vec{k} \times \vec{B}=0)$ parallel to the uniform background magnetic field $\vec{B}$. We extend earlier work [12, 13], who have studied weakly $\left(|\gamma| \ll \omega_{R}\right)$ damped and weakly amplified wave solutions, to the case of weakly propagating $\left(\omega_{R} \ll \gamma\right)$ solutions which includes in particular aperiodic fluctuations with $\omega_{R}=0$. $\omega_{R}$ and $\gamma$ here refer to real and imaginary part of the complex frquency $\omega=\omega_{R}+i \gamma$.

We start our investigation for equal mass thermal plasmas such as electron-positron pair plasmas which enormously facilitates the theoretical analysis due to the equality of the values of the electron and positron plasma and gyrofrequencies. However, a study of equal mass plasmas more than purely academic, as such plasmas [14] are relevant for the emission regions in active galactic nuclei [15], gamma-ray bursts [16, 17] and accreting magnetic degenerate dwarfs [18]. In future work we will investigate the case of electron-proton plasmas where more fluctuation modes result from the different mass ratio $\mu=m_{p} / m_{e}=1836$. In our studies we also adopt nonrelativistic plasma temperatures which is appropriate especially for the solar wind case.

\section{DISPERSION RELATIONS}

\subsection{Basic Equations}

For a nonzero background magnetic field strength the nonrelativistic dispersion relations for right-handed (RH) and left-handed $(\mathrm{LH})$ polarized fluctuations with wave vectors $\vec{k} \times \vec{B}=0$ in a thermal pair plasma are [19]

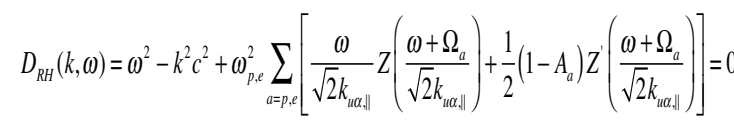

and

$D_{L H}(k, \omega)=\omega^{2}-k^{2} c^{2}+\omega_{p, e}^{2} \sum\left[\frac{\omega}{a=p, e}\left[\left(\frac{\omega-\Omega_{a}}{\sqrt{2} k_{u \alpha, \|}}\right)+\frac{1}{2}\left(1-A_{a}\right) Z\left(\frac{\omega-\Omega_{a}}{\sqrt{2} k_{u \alpha, \|}}\right)\right]=0\right.$

respectively, where we sum over a positron (p)-electron (e) pair plasma and where $k=\left|k_{\|}\right|$. $\omega_{p, e}$ denotes the electron plasma frequency, $u_{a, \|}=\left(k_{B} T_{a, \|} / m_{a}\right)^{1 / 2}$ is the parallel thermal velocity of component $a$, and $A_{a}=T_{a, \perp} / T_{a, \|}$ is the temperature anisotropy of component $a$, where the directional subscripts refer to directions relative to the background magnetic field. The dispersion relations (1) and (2) allow for different values of the positron and electron parallel temperatures and temperature anisotropies.

$Z(x)$ and $Z^{\prime}(x)$ denote the plasma dispersion function [20] and its derivative

$Z(x)=\pi^{-1 / 2} \int_{-\infty}^{\infty} d t \frac{e^{-t^{2}}}{t-x}$

with the well-known propertes

$$
Z^{\prime}(x)=-2[1+x Z(x)],
$$

and

$Z(-x)=2 \pi^{1 / 2} i e^{-x^{2}}-Z(x), \quad Z^{\prime}(-x)=4 \pi^{1 / 2} i x e^{-x^{2}}+Z^{\prime}(x)$

We will frequently use the asymptotic expansions

$Z(x) \simeq i \pi^{1 / 2} e^{-x^{2}}-2 x\left[1-\frac{2 x^{2}}{3}\right],|x| \ll 1$

and

$Z(x) \simeq i \sigma \pi^{1 / 2} e^{-x^{2}}-\frac{1}{x}\left[1+\frac{1}{2 x^{2}}+\frac{3}{4 x^{4}}\right],|x| \gg 1$

where $\sigma=0$ if $\mathfrak{I}(x)>0, \sigma=1$ if $\mathfrak{I}(x)=0$ and $\sigma=2$ if $\mathfrak{I}(x)<0$.

\subsubsection{Validity of Linear Dispersion Relation}

With the proper analytic continuation of the plasma dispersion function the dispersion relations (1) and (2) are valid in the whole complex frequency plane. However, the unstable solutions of the dispersion relations with positive $\gamma>0$ growth rates only hold during the linear phase of the instability, because the dispersion relations are derived from the linearized Vlasov equations under the assumption that the fluctuations in the electromagnetic fields $(|\delta \vec{E}|,|\delta \vec{B}|)$ and in the particle distribution function $\delta f$ and the electromagnetic fields are small compared to the unperturbed uniform magnetic field $B$ and distribution function $f_{0}$ taken here as bi-Maxwellians. In this case nonlinear terms in 
fluctuating quantities are negligible small compared to linear terms.

For any instability the fluctuating distribution function is given by its Fourier-transform

$$
\delta f(t)=\int d k M(k) e^{i \omega_{R}(k) t} e^{\gamma_{R}(k) t}
$$

Of particular interest in the instabilities investigated below, is the maximum growth rate $\gamma_{\max }$ at the wavenumber $k_{\text {max }}$, which allows us to approximate the fluctuating distribution function (8)) from above as

$\delta f(t) \leq e^{\gamma_{\max } t} \int d k M_{0}(k) e^{i \omega_{R}(k) t}$

Parseval's theorem then yields

$$
|\delta f(t)| \leq e^{\gamma_{\mathrm{max}} t}|\delta f(0)|,
$$

indicating that the fluctuating distribution function exponentially increases with time from its initial $(t=0)$ negligible value $\delta f(0)$.

The linear phase of the instability is defined by $|\delta f(t)| / f_{0}<1$, which with Eq. (10) corresponds to times

$0 \leq t \leq t_{L}, t_{L}=\gamma_{\max }^{-1} \ln \left(\frac{f_{0}}{|\delta f(0)|}\right)$

In this initial time interval $0 \leq t \leq t_{L}$, the fluctuating distribution function is negligible small compared to $f_{0}$, and the linear dispersion relations (1) and (2) can be used.

For later times $t>t_{L}$, the growing oscillations will completely modify the initial configuration $f_{0}$, so that the original ansatz of small fluctuations in the linearized Vlasov equation will become invalid. However, the calculation of the imaginary part of the frequency $\gamma$ of the allowed plasma modes provides the important information whether the chosen initial state $\left(f_{0}, \vec{B}_{0}\right)$ is stable $(\gamma<0)$ or unstable $(\gamma>0)$ to the growth of initially small perturbations. To what final configuration an unstable system develops cannot be answered by this investigation of linear stability.

\subsubsection{Introduction of Phase Speeds}

It is convenient to introduce the complex phase speeds

$f=\frac{\omega}{k c}=\frac{\omega_{R}+i \gamma}{k c}=R+i S, \quad R=\frac{\omega_{R}}{k c}, \quad S=\frac{\gamma}{k c}$,

and

$b=\Omega_{p} k c, \quad w=\omega_{p, e} k c$

where $\Omega_{p}=e B / m_{e} c$ is the positive positron gyrofrequency, and the dimensionless positron and electron temperatures

$\Theta_{p} \equiv\left(\frac{2 k_{B} T_{p, \|}}{m_{e} c^{2}}\right)^{1 / 2}, \Theta_{e} \equiv\left(\frac{2 k_{B} T_{e, \|}}{m_{e} c^{2}}\right)^{1 / 2}$
Throughout this work, in the classification of Swanson [21] we discuss high density plasmas with $\omega_{p, e} \gg \Omega_{p}$, corresponding to $w \gg b$ which applies to nearly all astrophysical plasmas. These plasmas are dense enough that the electron plasma frequency is much larger than the electron gyrofrequency, but small enough that elastic Coulomb collisions can be neglected: the small values of the plasma parameter $g=3 v_{e e} / \omega_{p, e}<O\left(10^{-10}\right)$ in astrophysical plasmas calculated in table 8.1 of Schlickeiser [1] indicate that the elastic Coulomb collision frequency is more than 10 orders of magnitude smaller than the electron plasma frequency. Thus astrophysical plasmas are effectively collision-free on the shortest time and length scales.

The two dispersion relations (1) and (2) then read

$$
\begin{aligned}
& 0=\frac{D_{R H}(k, f)}{k^{2} c^{2}}=\Lambda_{R H}(k, f)=f^{2}-1+ \\
& w^{2}\left[\begin{array}{c}
\frac{f}{\Theta_{p}} Z\left(\frac{f+b}{\Theta_{p}}\right)+\frac{1}{2}\left(1-A_{p}\right) Z^{\prime}\left(\frac{f+b}{\Theta_{p}}\right) \\
+\frac{f}{\Theta_{e}} Z\left(\frac{f-b}{\Theta_{e}}\right)+\frac{1}{2}\left(1-A_{e}\right) Z^{\prime}\left(\frac{f-b}{\Theta_{e}}\right)
\end{array}\right] \\
& =f^{2}-1-\frac{w^{2}}{2}\left[4+A_{p} Z^{\prime}\left(\frac{f+b}{\Theta_{p}}\right)+A_{e} Z^{\prime}\left(\frac{f-b}{\Theta_{e}}\right)\right] \\
& +w^{2} b\left[\frac{1}{\Theta_{e}} Z\left(\frac{f-b}{\Theta_{e}}\right)-\frac{1}{\Theta_{p}} Z\left(\frac{f+b}{\Theta_{p}}\right)\right]
\end{aligned}
$$

and

$$
\begin{aligned}
& 0=\frac{D_{L H}(k, f)}{k^{2} c^{2}}=\Lambda_{L H}(k, f)=f^{2}-1+ \\
& w^{2}\left[\begin{array}{l}
\frac{f}{\Theta_{p}} Z\left(\frac{f-b}{\Theta_{p}}\right)+\frac{1}{2}\left(1-A_{p}\right) Z^{\prime}\left(\frac{f-b}{\Theta_{p}}\right)+\frac{f}{\Theta_{e}} Z\left(\frac{f+b}{\Theta_{e}}\right) \\
+\frac{1}{2}\left(1-A_{e}\right) Z^{\prime}\left(\frac{f+b}{\Theta_{e}}\right)
\end{array}\right] \\
& =f^{2}-1-\frac{w^{2}}{2}\left[4+A_{p} Z^{\prime}\left(\frac{f-b}{\Theta_{p}}\right)+A_{e} Z^{\prime}\left(\frac{f+b}{\Theta_{e}}\right)\right] \\
& +w^{2} b\left[\frac{1}{\Theta_{p}} Z\left(\frac{f-b}{\Theta_{p}}\right)-\frac{1}{\Theta_{e}} Z\left(\frac{f+b}{\Theta_{e}}\right)\right]
\end{aligned}
$$

where we have used Eq. (4). We immediately notice the symmetry $\Lambda\left(-k_{\|}, f\right)=\Lambda\left(k_{\|}, f\right)$ of both dispersion relations allowing to consider only positive values of the wavenumber $k>0$.

The dispersion relations (15) and (16) can be separated into real and imaginary parts

$\Lambda(R, S)=\Re \Lambda(R, S)+i \mathfrak{I} \Lambda(R, S)=0$ 
implying the two conditions

$\Re \Lambda(R, S)=0, \mathfrak{I} \Lambda(R, S)=0$

For a wide class of initial distribution functions, including the bi-Maxwellian distribution, the dispersion relation $\Lambda(R, S)$ is a meromorphic function of the complex variable $f=R+i S$. This allows us to Taylor-expand the dispersion relation around different ponits in the complex plane. We consider two expansions in turn which are referred to as weak damping/amplification limit and weak propagation limit, respectiverly.

\subsection{Weak Damping/Amplification Limit}

In the weak damping approximation we equate the real and imaginary parts to zero (see Eq. (18)), and make a Taylor-expansion around $S=0$ to obtain

$$
\Re \Lambda(R, S) \simeq \Re \Lambda(R, S=0)+S\left[\frac{\partial \Re \Lambda(R, S)}{\partial S}\right]_{S=0}=0,
$$

and

$$
\mathfrak{I} \Lambda(R, S) \simeq \mathfrak{I} \Lambda(R, S=0)+S\left[\frac{\partial \mathfrak{I} \Lambda(R, S)}{\partial S}\right]_{S=0}=0,
$$

Since $\Lambda(R, S)$ is a meromorphic function of the complex variable $f=R+i S$, we may use the Cauchy-Riemann relations

$\frac{\partial \Re \Lambda(R, S)}{\partial R}=\frac{\partial \mathfrak{I} \Lambda(R, S)}{\partial S}, \frac{\partial \Re \Lambda(R, S)}{\partial S}=-\frac{\partial \mathfrak{I} \Lambda(R, S)}{\partial R}$

Eq. (20) then indicates that $\mathfrak{I} \Lambda$, to first order in $S$ is

$$
\mathfrak{I} \Lambda(R, S) \simeq \mathfrak{I} \Lambda(R, S=0)+S \frac{\partial \mathfrak{R} \Lambda(R, S=0)}{\partial R}=0
$$

Likewise, Eq. (19) yields

$$
\Re \Lambda(R, S=0)-S \frac{\partial \mathfrak{I} \Lambda(R, S=0)}{\partial R}=0
$$

which, in combination with Eq. (22), yields to lowest order in the small quantity $(S / R)^{2} \ll 1$ that the real part of the dispersion relation satisfies

$\Re \Lambda(R, S=0)=0$

Eq. (22) provides the corresponding imaginary part

$$
S=-\frac{\mathfrak{I} \Lambda(R, S=0)}{\frac{\partial \Re \Lambda(R, S=0)}{\partial R}}
$$

in the weak damping and amplification limit. For consistency, the resulting weak damping/amplification solutions have to fulfil $|S| \ll R$, which has to be checked aposteriori. According to Eq. (11) the weak damping and amplification solutions are valid for times

$$
0 \leq t \leq \frac{k_{\max } c}{S_{\max }} \ln \left(\frac{f_{0}}{|\delta f(0)|}\right) .
$$

\subsection{Weak Propagation Limit}

In the weak propagation limit $(|S| \gg R)$ we Taylorexpand around $R=0$ to obtain

$\mathfrak{R} \Lambda(R, S) \simeq \Re(R=0, S)+R\left[\frac{\partial \mathfrak{R} \Lambda(R, S)}{\partial R}\right]_{R=0}=0$,

and

$\mathfrak{I} \Lambda(R, S) \simeq \mathfrak{I} \Lambda(R=0, S)+R\left[\frac{\partial \mathfrak{I} \Lambda(R, S)}{\partial R}\right]_{R=0}=0$

The Cauchy-Riemann relations (21) then indicate that to first order in $R$

$\Re \Lambda(R=0, S)+R\left[\frac{\partial \mathfrak{I} \Lambda(R=0, S)}{\partial S}\right]=0$,

and

$\mathfrak{I} \Lambda(R=0, S)-R\left[\frac{\partial \Re \Lambda(R=0, S)}{\partial S}\right]=0$

Inserting Eq. (30) into Eq. (29), we find that to lowest order in the small quantity $(R /|S|)^{2} \ll 1$ the real part of the dispersion relation satisfies

$\Re \Lambda(R=0, S)=0$

whereas Eq. (30) provides the corresponding real part

$R=\frac{\mathfrak{I} \Lambda(R=0, S)}{\frac{\partial \mathfrak{R} \Lambda(R=0, S)}{\partial S}}$

in the weak propagation limit. For consistency, the resulting weak propagation solutions have to fulfil $R \ll|S|$, which has to be checked aposteriori. According to Eq. (11) the weakly propagating solutions are valid for times

$0 \leq t \leq \frac{k_{\max } c}{S_{\max }} \ln \left(\frac{f_{0}}{|\delta f(0)|}\right)$.

\subsection{Kinetic and Non-Kinetic Regimes}

In terms of the complex phase speed $f=R+i S$ the real and imaginary parts of the two dispersion relations (15) and (16) read

$$
\begin{aligned}
& 0=\Re \Lambda_{R H}(R, S)=R^{2}-S^{2}-1-\frac{w^{2}}{2}\left[4+A_{p} \Re Z^{\prime}\left(\frac{R+i S+b}{\Theta_{p}}\right)+A_{e} \Re Z^{\prime}\left(\frac{R+i S-b}{\Theta_{e}}\right)\right] \\
& +w^{2} b\left[\frac{1}{\Theta_{e}} \mathfrak{R}\left(\frac{R+i S-b}{\Theta_{e}}\right)-\frac{1}{\Theta_{p}} \mathfrak{R} Z\left(\frac{R+i S+b}{\Theta_{p}}\right)\right], \\
& 0=\mathfrak{I} \Lambda_{R H}(R, S)=2 R S-\frac{w^{2}}{2}\left[A_{p} \mathfrak{I} Z^{\prime}\left(\frac{R+i S+b}{\Theta_{p}}\right)+A_{e} \mathfrak{I} Z^{\prime}\left(\frac{R+i S-b}{\Theta_{e}}\right)\right] \\
& +w^{2} b\left[\frac{1}{\Theta_{e}} \mathfrak{I Z}\left(\frac{R+i S-b}{\Theta_{e}}\right)-\frac{1}{\Theta_{p}} \mathfrak{I Z}\left(\frac{R+i S+b}{\Theta_{p}}\right)\right],
\end{aligned}
$$

and 


$$
\begin{aligned}
& 0=\Re \Lambda_{L H}(R, S)=R^{2}-S^{2}-1-\frac{w^{2}}{2}\left[4+A_{p} \Re Z^{\prime}\left(\frac{R+i S-b}{\Theta_{p}}\right)+A_{e} \Re Z^{\prime}\left(\frac{R+i S+b}{\Theta_{e}}\right)\right] \\
& +w^{2} b\left[\frac{1}{\Theta_{p}} \Re Z\left(\frac{R+i S-b}{\Theta_{p}}\right)-\frac{1}{\Theta_{e}} \Re Z\left(\frac{R+i S+b}{\Theta}\right)\right] \text {, } \\
& 0=\mathfrak{I} \Lambda_{L H}(R, S)=2 R S-\frac{w^{2}}{2}\left[A_{p} \mathfrak{I} Z^{\prime}\left(\frac{R+i S-b}{\Theta_{p}}\right)+A_{e} \mathfrak{I} Z^{\prime}\left(\frac{R+i S+b}{\Theta_{e}}\right)\right] \\
& +w^{2} b\left[\frac{1}{\Theta_{p}} \mathfrak{I Z}\left(\frac{R+i S-b}{\Theta_{p}}\right)-\frac{1}{\Theta_{e}} \mathfrak{I Z}\left(\frac{R+i S+b}{\Theta_{e}}\right)\right]
\end{aligned}
$$

In the following we will simplify the analysis by considering only equal parallel temperature plasmas $\Theta_{p}=\Theta_{e}=\Theta$.

We will analyze Eqs. (34)-(37) in the limits of weak and strong damping/amplification for small and large absolute values of the arguments

$$
\left|P_{ \pm}\right|=\left|\frac{R+i S \pm b}{\Theta}\right|=\frac{\sqrt{(R \pm b)^{2}+S^{2}}}{\Theta},
$$

resulting in a separation of the complex phase speed $(R-S)$-plane into different regions with either $\left|P_{ \pm}\right| \gg 1$ or $\left|P_{ \pm}\right| \ll 1$, as illustrated in Fig. (1). The condition $\left|P_{ \pm}\right|=1$ separates these regions and corresponds to

$$
(R \pm b)^{2}+S^{2}=\Theta^{2}
$$

which defines circles within the $(R-S)$-plane with radii $\Theta$. For $R$ and $S$ values within the circles the asymptotics (6) of the plasma dispersion function. can be applied. Alternatively, for $R$ and $S$ values outside the circles on has to use the asymptotics (7) of the plasma dispersion function.

The separation condition $\left|P_{ \pm}\right|=1$ depends strongly on the ratio $b / \Theta$. The case $b \gg \Theta$ of strong magnetic fields is referred to as kinetic regime, whereas the opposite case $b \ll \Theta$ is referred to as non-kinetic regime. Because

$\frac{b}{\Theta}=\frac{1}{k \rho}=\frac{2 \pi \lambda}{\rho}$

where

$\rho=\frac{\sqrt{2 k_{B} T_{\|} / m_{e}}}{\Omega_{p}}$,

denotes the thermal pair gyroradius, the kinetic regime corresponds to small $(\rho \ll \lambda)$ ratios of the pair thermal pair gyroradius to the wavelength of the fluctuatiobns. In the nonkinetic regime, the thermal pair gyrodius is large compared to the wavelength. Geometrically, in the kinetic regime the positron and electron circles in Fig. (1) do not overlap, whereas in the non-kinetic regime they overlap.

In the respective limits of large or small absolute values of the arguments of the plasma dispersion function both in the weak and strong damping/amplification approximation we will obtain formal mathematical solutions for $R$ and $S$. These solutions are only acceptable if they indeed fulfil the initially made assumptions on weak or strong damping/ amplification which has to be checked a posteriori. Because the solutions depend in particular on the kineticity ratio $(b / \Theta)$, it is convenient to introduce the parallel plasma beta

$\beta_{\|}=\frac{\Theta^{2} w^{2}}{b^{2}}=\frac{8 \pi n_{e} k_{B} T_{\|}}{B^{2}}$

which expresses the magnetic field strength as

$b=\frac{\Theta w}{\beta_{\|}^{1 / 2}}$

in terms of the electron plasma frequency $(w)$, the parallel temperature $(\Theta)$ and the parallel plasma beta $\beta_{\|}$. Our restriction to only high-density plasmas with $\omega_{p, e}>\Omega_{p}$ or $w>b$ then requires to have parallel plasma betas $\beta_{\|}>\Theta^{2}$.

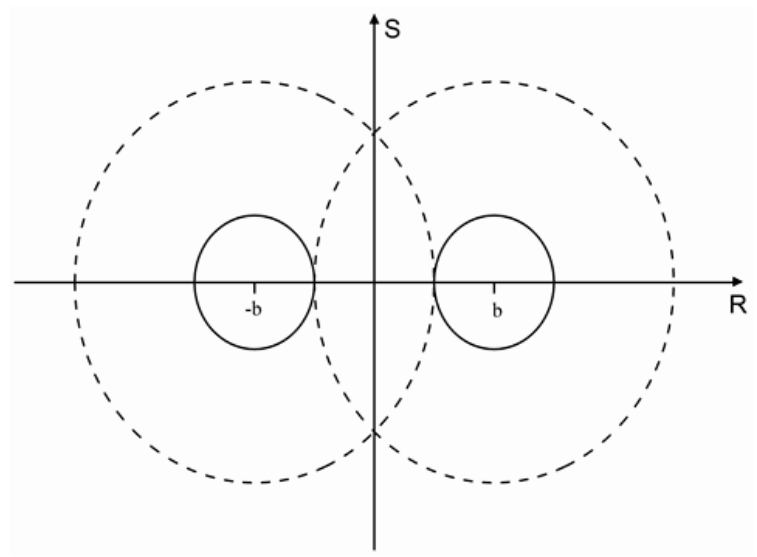

Fig. (1). Separation of the complex phase speed plane into regions where the absolute value of the argunments of the plasma dispersion function $\left|P_{ \pm}\right|=\left|\frac{R+i S \pm b}{\Theta}\right|=\sqrt{(R \pm b)^{2}+S^{2}} / \Theta$ is small and large compared to unity. Within the concentric circles around $\pm b$ with radius $\Theta$ the arguments are small compared to unity; outside the circles they are large compared to unity. A value of $b=2$ is adopted. The intersecting dashed circles refer to the case $\Theta=3>b$; the non-intersecting circles refer to the case $\Theta=1<b$.

Likewise, the resulting solutions $R\left(\beta_{\|}, w, \Theta\right)$ and $S\left(\beta_{\|}, w, \Theta\right)$ depend on these three basic plasma parameterrs. Note that the wavenumber dependence is included in $w$.

\section{WEAKLY DAMPED AND \\ SOLUTIONS}

AMPLIFIED

For weakly damped or amplified fluctuations Eqs. (34)(37) read

$$
\begin{aligned}
& 0=\Re \Lambda_{R H}(R, S=0)=R^{2}-1-\frac{w^{2}}{2}\left[4+A_{p} \Re Z^{\prime}\left(\frac{R+b}{\Theta}\right)+A_{e} \Re Z^{\prime}\left(\frac{R-b}{\Theta}\right)\right] \\
& +\frac{w^{2} b}{\Theta}\left[\Re Z\left(\frac{R-b}{\Theta}\right)-\Re Z\left(\frac{R+b}{\Theta}\right)\right],
\end{aligned}
$$




$$
\begin{aligned}
\mathfrak{I} \Lambda_{R H}(R, S=0)= & -\frac{w^{2}}{2}\left[A_{p} \mathfrak{I} Z^{\prime}\left(\frac{R+b}{\Theta}\right)+A_{e} \mathfrak{I} Z^{\prime}\left(\frac{R-b}{\Theta}\right)\right] \\
& +\frac{w^{2} b}{\Theta}\left[\mathfrak{Z}\left(\frac{R-b}{\Theta}\right)-\mathfrak{I Z}\left(\frac{R+b}{\Theta}\right)\right]
\end{aligned}
$$

and

$$
\begin{aligned}
& 0=\Re \Lambda_{L H}(R, S=0)=R^{2}-1-\frac{w^{2}}{2}\left[4+A_{p} \Re Z^{\prime}\left(\frac{R-b}{\Theta}\right)+A_{e} \Re Z^{\prime}\left(\frac{R+b}{\Theta}\right)\right] \\
& +\frac{w^{2} b}{\Theta}\left[\Re Z\left(\frac{R-b}{\Theta}\right)-\Re Z\left(\frac{R+b}{\Theta}\right)\right] \text {, } \\
& \mathfrak{I} \Lambda_{L H}(R, S=0)=-\frac{w^{2}}{2}\left[A_{p} \mathfrak{I} Z^{\prime}\left(\frac{R-b}{\Theta}\right)+A_{e} \mathfrak{I} Z^{\prime}\left(\frac{R+b}{\Theta}\right)\right] \\
& +\frac{w^{2} b}{\Theta}\left[\mathfrak{I Z}\left(\frac{R-b}{\Theta}\right)-\mathfrak{I Z}\left(\frac{R+b}{\Theta}\right)\right],
\end{aligned}
$$

which have to be investigated for positive values of $R \geq 0$.

As noted before, these dispersion relations can be further reduced with the asymptotic expansions (6) and (7), depending on the absolute values of the arguments

$X_{1}=\frac{|R+b|}{\Theta}, X_{2}=\frac{|R-b|}{\Theta}$

of the plasma dispersion function and its derivative being small or large compared to unity. Expressed in terms of the plasma beta (43) the conditions $X_{1,2}=1$ correspond to

$$
R 1=\Theta\left(1-w \beta_{\|}^{-1 / 2}\right)
$$

for $X_{1}=1$, which is automatically fulfilled for $\beta_{\|} \leq w^{2}$. Likewise, we obtain for $X_{2}=1$ in the case $R<b$ that

$R 2(R<b)=\Theta\left(w \beta_{\|}^{-1 / 2}-1\right)$

and

$$
R 3(R>b)=\Theta\left(1+w \beta_{\|}^{-1 / 2}\right)
$$

for $R>b$. As illustrated in Fig. (2) the three curves $R 1\left(\beta_{\|}\right), R 2\left(\beta_{\|}\right)$and $R 3\left(\beta_{\|}\right)$define dividing lines in the $\left(R, \beta_{\|}\right)$-solution plane that separate the regions where $X_{1,2}$ are larger or smaller than unity. For orientation we also plot the $b\left(\beta_{\|}\right)$-dependence (43) that indicates the location of the pair cyclotron phase speed $b$ in this diagram.

As indicated in Fig. (2) four different regions can be identified:

(1) The region WI well below the curve R2, where both $X_{1,2} \gg 1$. In this region the real phase speeds have to smaller than $R \ll b-\Theta$ while the plasma beta is restricted to values of $\Theta^{2} \beta_{\|} \ll w^{2}$, where the lower limt results from the high-density limit $b \ll w$.

(2) The region WII well above the curve R3 at all plasma betas $\beta_{\|} \gg \Theta^{2}$, where also both $X_{1,2} \ll 1$.
(3) The region WIII well below the curve R3 at all plasma betas and well above the curve R2 (for $\beta_{\|}<w^{2}$ ) and $\mathrm{R} 1$ (for $\beta_{\|}>w^{2}$ ). In this region $X_{1} \gg 1$ but $X_{2} \ll 1$.

(4) The region WIV at plasma betas $\beta_{\|}>w^{2}$ below the curve R1, where both $X_{1,2} \ll 1$.

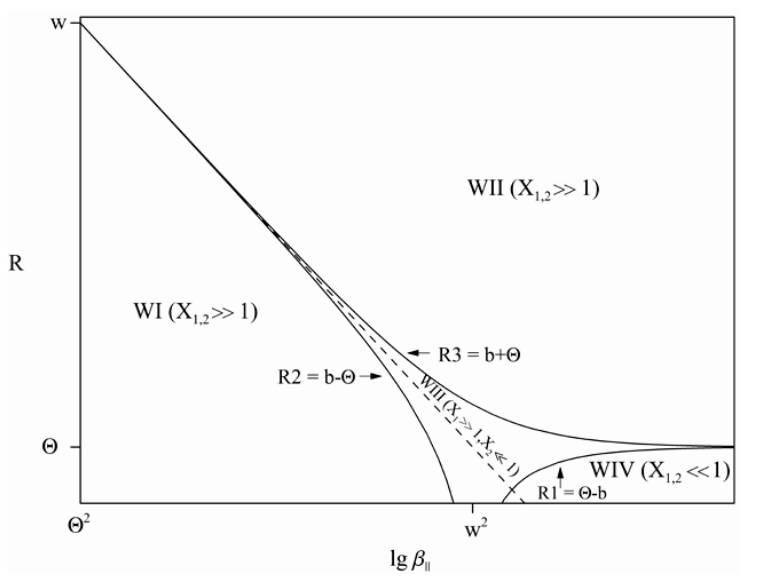

Fig. (2). Separation of the $R-\beta_{\|}$-parameter plane into four different regions depending on the values of $X_{1,2}$ being small or large compared to unity. $X_{1,2}$ are the arguments of the plasma dispersion function in the weak damping/amplification limit. For orientation the dashed curve shows the $b\left(\beta_{\|}\right)$-dependence ((43)) that indicates the location of the pair cyclotron phase speed $b$ in this diagram

The regions WIII and WIV need not to be discussed because it is well known $[22,23]$ that solutions with either or both $X_{1,2} \ll 1$ have $|S| \gg R$, so that the weak damping approximation cannot be applied here.

Pair Alfven and cyclotron wave solutions in anisotropic bi-Maxwellian electron-positron plasmas were investigating before by Gary and Karimabadi [13] by numerically solving the linear dispersion relation for fixed values of the temperature anisotropy and the plasma beta, with no restriction on the wave propagation direction, so they also include the case of parallel propagation considered here. Here we concentrate on the analytical derivation of the existence and instability conditions of parallel propagating pair Alfven and cyclotron waves.

\subsection{Imaginary Part of Weak Damping/Amplification Dispersion Relation}

Before considering the regions WI and WII in more detail we note that both asymptotic expansions (6) and (7) yield the same imaginary part of the dispersion relation

$$
\begin{aligned}
& 0=I \Lambda_{R H, H}(R, S=0)=\pi^{12} w^{2} \frac{b}{\Theta}\left[A_{p}\left[\frac{R}{b} \pm 1\right] e^{-\left(\frac{R \pm b}{\Theta}\right)^{2}}+A_{e}\left[\frac{R}{b} \mp 1\right] e^{-\left(\frac{R \mp b}{\Theta}\right)^{2}}+e^{-\left(\frac{R-b}{\Theta}\right)^{2}}-e^{-\left(\frac{R+b}{\Theta}\right)^{2}}\right) \\
& =\pi^{12} w^{2} \frac{b}{\Theta}\left(\left[A_{p, e}-1\right] e^{-\left(\frac{R+b}{\Theta}\right)^{2}}+\left[1-A_{e, p}\right] e^{-\left(\frac{R-b}{\Theta}\right)^{2}}+\frac{R}{b}\left[A_{p, e} e^{-\left(\frac{R+b}{\Theta}\right)^{2}}+A_{e, p} e^{-\left(\frac{R-b}{\Theta}\right)^{2}}\right]\right),
\end{aligned}
$$


irrespective of the values of $X_{1,2}$. For the same positron and electron anisotropy $\left(A_{p}=A_{e}=A_{0}\right)$ we obtain

$$
\begin{aligned}
& 0=\mathfrak{I} \Lambda_{R H, L H}\left(R, S=0, A_{0}\right) \\
& =-2 \pi^{1 / 2} \frac{b}{\Theta} w^{2} e^{-\frac{R^{2}+b^{2}}{\Theta^{2}}} \cosh \left(\frac{2 b R}{\Theta^{2}}\right) \\
& {\left[\left(A_{0}-1\right) \tanh \left(\frac{2 b R}{\Theta^{2}}\right)-\frac{A_{0} R}{b}\right]}
\end{aligned}
$$

3.2 Regions WI and WII: Pair Alfven Waves, Cyclotron Waves and Electromagnetic Light

In the regions WI and WII both $X_{1,2} \gg 1$, so that with

$\mathfrak{R}\left(\frac{R \pm b}{\Theta}\right) \simeq-\frac{\Theta}{R \pm b}\left[1+\frac{\Theta^{2}}{2(R \pm b)^{2}}\right]$

and

$\mathfrak{R} Z^{\prime}\left(\frac{R \pm b}{\Theta}\right) \simeq+\frac{\Theta^{2}}{(R \pm b)^{2}}\left[1+\frac{3 \Theta^{2}}{2(R \pm b)^{2}}\right]$

we obtain for the real parts of the dispersion relations (44) and (46) to lowest order in $\Theta^{2} \ll 1$

$0=\Re \Lambda_{R H, L H}(R, S=0)$

$=R^{2}-1+w^{2}\left[\frac{2 R^{2}}{b^{2}-R^{2}}+\frac{b^{2} \Theta^{2}\left(b^{2}+3 R^{2}\right)}{\left(b^{2}-R^{2}\right)^{3}}-\frac{A_{p} \Theta^{2}}{2(R \pm b)^{2}}-\frac{A_{e} \Theta^{2}}{2(R \mp b)^{2}}\right]$

In different limits the solutions of Eq. (56) describe pair Alfven waves, pair cyclotron waves and electromagnertic light. We consider each case in the next subsections.

3.3. Pair Alfven Waves at Phase Speeds $R \ll R 2<b$ for $\Theta^{2} \ll \beta_{\|}<w^{2}$

For phase speeds $R \ll b$ the dispersion relation (56) simplifies to

$$
\begin{aligned}
& 0=\Re \Lambda_{R H, L H}(R, S=0)=R^{2}\left(1+\frac{2 w^{2}}{b^{2}}\right) \\
& -1+w^{2} \frac{\Theta^{2}}{b^{2}}\left[\left(1-\frac{A_{p}+A_{e}}{2}\right)\right. \\
& \left. \pm\left(A_{p}-A_{e}\right) \frac{R}{b}+\left(6-\frac{3\left(A_{p}+A_{e}\right)}{2}\right) \frac{R^{2}}{b^{2}}\right] \\
& =R^{2}\left(1+\frac{2 w^{2}}{b^{2}}\left(1+\frac{3(2-A) \Theta^{2}}{2}\right)\right) \pm \beta_{\|}\left(A_{p}-A_{e}\right) \frac{R}{b}-\left[1+(A-1) \beta_{\|}\right] \\
& \simeq\left(1+\frac{c^{2}}{V_{e}^{2}}\right) R^{2} \pm \beta_{\|}\left(A_{p}-A_{e}\right) \frac{R}{b}-\left[1+(A-1) \beta_{\|}\right]
\end{aligned}
$$

where we introduce the pair Alfven speed

$$
\frac{2}{b^{2}} w^{2}=\frac{8 \pi n_{e} m_{e} c^{2}}{B^{2}}=\frac{c^{2}}{V_{e}^{2}},
$$

the parallel pair plasma beta (42) and the combined plasma temperature anisotropy

$A=\frac{A_{p}+A_{e}}{2}$

For high-density plasmas $V_{e} \ll c$, the dispersion relation (57) yields the LH and RH polarized pair Alfven modes with the same

$$
\begin{aligned}
& R \simeq \frac{b}{\sqrt{2} w} \sqrt{1+(A-1) \beta_{\|}} \\
& =\Theta \sqrt{\frac{1+(A-1) \beta_{\|}}{2 \beta_{\|}}}=\frac{V_{e}}{c} \sqrt{1+(A-1) \beta_{\|}},
\end{aligned}
$$

which either can propagate forward and backward. Note that the condition $R \gg b$ requires $w \gg 2^{-1 / 2}$. Moreover, these four pair Alfven modes only exist for temperature anisotropies such that $1+(A-1) \beta_{\|} \geq 0$ corresponding to

$A>\left(1-\frac{1}{\beta_{\|}}\right)$

which includes the isotropic $\left(A_{p}=A_{e}=A=1\right)$ plasma temperature case. For small plasma betas $\left(\beta_{\|} \leq 1\right)$ the condition (61) is always fulfilled whereas for large plasma betas $\left(\beta_{\|}>1\right)$ the combined anisotropy $A$ has to be larger than $1-\beta_{\|}^{-1}$.

Eq. (57) also provides

$$
\frac{\partial \Re \Lambda_{R H, L H}(R, S=0)}{\partial R}=2\left(1+\frac{c^{2}}{V_{e}^{2}}\right) R \pm \frac{\beta_{\|}\left(A_{p}-A_{e}\right)}{b} \simeq 2 \frac{c^{2} V_{e}^{2}}{R},
$$

so that for all four modes according to Eqs. (25) and (52) the growth/damping rate is

$$
\begin{aligned}
& S_{R H, L H}=-\frac{V_{e}^{2}}{2 c^{2} R} \mathfrak{I} \Lambda_{R H, L H}(R, S=0)= \\
& -\frac{\pi^{1 / 2} b^{3}}{4 \Theta R}\left(\begin{array}{l}
A_{p}\left[\frac{R}{b} \pm 1\right] e^{-\left(\frac{R \pm b}{\Theta}\right)^{2}} \\
\left.+A_{e}\left[\frac{R}{b} \mp 1\right] e^{-\left(\frac{R \mp b}{\Theta}\right)^{2}}+e^{-\left(\frac{R-b}{\Theta}\right)^{2}}-e^{-\left(\frac{R+b}{\Theta}\right)^{2}}\right)
\end{array}\right.
\end{aligned}
$$

For isotropic $\left(A_{p}=A_{e}=1\right)$ plasma temperatures all four Alfven modes are damped with the rate

$$
S_{R H, L H}\left(A_{p}=A_{e}=1\right)=-\frac{\pi^{1 / 2} b^{2}}{2 \Theta} e^{-\frac{R^{2}+b^{2}}{\Theta^{2}}} \cosh \left(\frac{2 b R}{\Theta^{2}}\right)
$$

For the same positron and electron anisotropy ( $A_{p}=A_{e}=A_{0}$ ) the growth $/$ damping rate (63) reads

$$
\begin{aligned}
& S_{R H, L H}\left(A_{p}=A_{e}=A_{0}\right)=\frac{\pi^{1 / 2} b^{3}}{2 \Theta R} e^{-\frac{R^{2}+b^{2}}{\Theta^{2}}} \cosh \left(\frac{2 b R}{\Theta^{2}}\right) \\
& {\left[\left(A_{0}-1\right) \tanh \left(\frac{2 b R}{\Theta^{2}}\right)-\frac{A_{0} R}{b}\right]}
\end{aligned}
$$


We immediately see that for $A_{0} \leq 1$ this rate is always negative. In order to drive the pair Alfven modes unstable values with $A_{0}>1$ are required such that

$$
\left(A_{0}-1\right) \tanh \left(\frac{2 b R}{\Theta^{2}}\right)>\frac{A_{0} R}{b},
$$

\subsubsection{Pair Alfven Wave Instability Condition}

The tanh-function is well approximated by

$$
\tanh (x) \simeq \frac{x}{1+x}
$$

yielding for condition (66)

$$
\frac{A_{0}-1}{A_{0}}>\frac{\beta_{\|}}{2 w^{2}}+\frac{R}{b}
$$

Insertion of the solution (60) yields for the instability condition

$$
\frac{A_{0}-1}{A_{0}}>\frac{\beta_{\|}}{2 w^{2}}+\sqrt{\frac{1+\left(A_{0}-1\right) \beta_{\|}}{2 w^{2}}},
$$

which defines a quadratic inequality for $\beta_{\|}$in terms of $A_{0}$. Scaling $\beta_{\|}=2 w^{2} B$ with $B<(1 / 2)$ the condition (69) reads

$$
\frac{A_{0}-1}{A_{0}}-B>\sqrt{\frac{1}{2 w^{2}}+\left(A_{0}-1\right) B}
$$

Because the right hand side of this inequality is positive, we derive as first constraint that $B<\left(A_{0}-1\right) / A_{0}$ or

$$
\beta_{\|}<2 w^{2} \frac{A_{0}-1}{A_{0}},
$$

corresponding to the condition

$$
A_{0}>\frac{2 w^{2}}{2 w^{2}-\beta_{\|}}
$$

and $\beta_{\|}<2 w^{2}$. The solution of inequality (70) with the requirement $((71))$ is

$$
B=\frac{\beta_{\|}}{2 w^{2}}>\frac{A_{0}-1}{A_{0}}\left[\frac{A_{0}+2}{2}-\sqrt{\left(\frac{A_{0}+2}{2}\right)^{2}-1+\left(\frac{A_{0}}{A_{0}-1}\right)^{2} \frac{1}{2 w^{2}}}\right],
$$

In order for this to be positive we need as second condition that $A_{0} /\left(A_{0}-1\right)<2^{1 / 2} w$ or

$$
A_{0}>\frac{\sqrt{2} w}{\sqrt{2} w-1},
$$

restricting the plasma frequency phase speeds to values $w>1 / \sqrt{2}$. Pair Alfven waves can only be driven unstable at wavenumbers smaller than $k<\sqrt{2} \omega_{p, e} / c$.

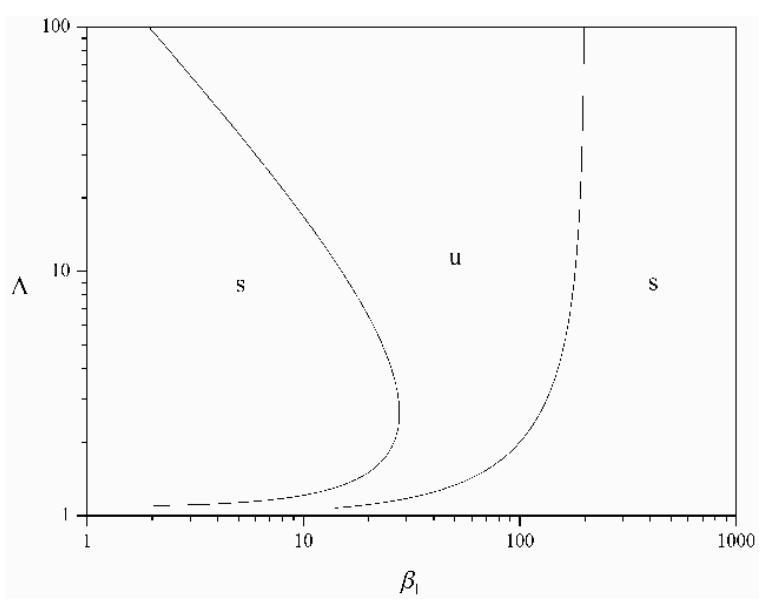

Fig. (3). Anisotropy diagram for pair Alfven waves. Stable regions are marked by "s", unstable regions are marked by"u". The pair Alfven wave instability occurs for values of $A>1$ for anisotropies $A$ and parallel plasma beta values $\beta_{\|}$located between the left and right curve. A value of $w=10$ is adopted.

The three instability conditions (72), (73) and (74) are illustrated in Fig. (3). Pair Alfven wave instability is possible for anisotropies $A$ and parallel plasma beta values $\beta_{\|}$located between the left and right curve:

(1) for plasma betas $\beta_{\|} \leq \sqrt{2} w$ it is required that $A_{0}>\sqrt{2} w /(\sqrt{2} w-1)$ and that

$$
\beta_{\|}>2 w^{2} \frac{A_{0}-1}{A_{0}}\left[\frac{A_{0}+2}{2}-\sqrt{\left(\frac{A_{0}+2}{2}\right)^{2}-1+\left(\frac{A_{0}}{A_{0}-1}\right)^{2} \frac{1}{2 w^{2}}}\right](75)
$$

(2) for plasma betas $\sqrt{2} w<\beta_{\|}<2 w^{2}$ the instability condition is

$$
\begin{aligned}
& 2 w^{2} \frac{A_{0}-1}{A_{0}}\left[\frac{A_{0}+2}{2}-\sqrt{\left(\frac{A_{0}+2}{2}\right)^{2}-1+\left(\frac{A_{0}}{A_{0}-1}\right)^{2} \frac{1}{2 w^{2}}}\right] \\
& <\beta_{\|}<2 w^{2} \frac{A_{0}-1}{A_{0}}
\end{aligned}
$$

No instability occurs in high-beta plasmas $\beta_{\|}>2 w^{2}$ and pair plasma frequency phase speeds $w<1 / \sqrt{2}$.

Because of condition (74) the requirement (73) with $g=\sqrt{2} w>1$ can be well approximated by

$$
\begin{aligned}
& \left.B=\frac{\beta_{\|}}{g^{2}} \geq \frac{A_{0}-1}{A_{0}\left(A_{0}+2\right.}\right)\left[1-\frac{A_{0}^{2}}{g^{2}\left(A_{0}-1\right)^{2}}\right] \\
& =\frac{\frac{g^{2}-1}{g^{2}}\left(A_{0}-\frac{g}{g-1}\right)\left(A_{0}-\frac{g}{g+1}\right)}{A_{0}\left(A_{0}+2\right)\left(A_{0}-1\right)}
\end{aligned}
$$


Table 1. Properties of Weakly Amplified Pair Alfven Waves

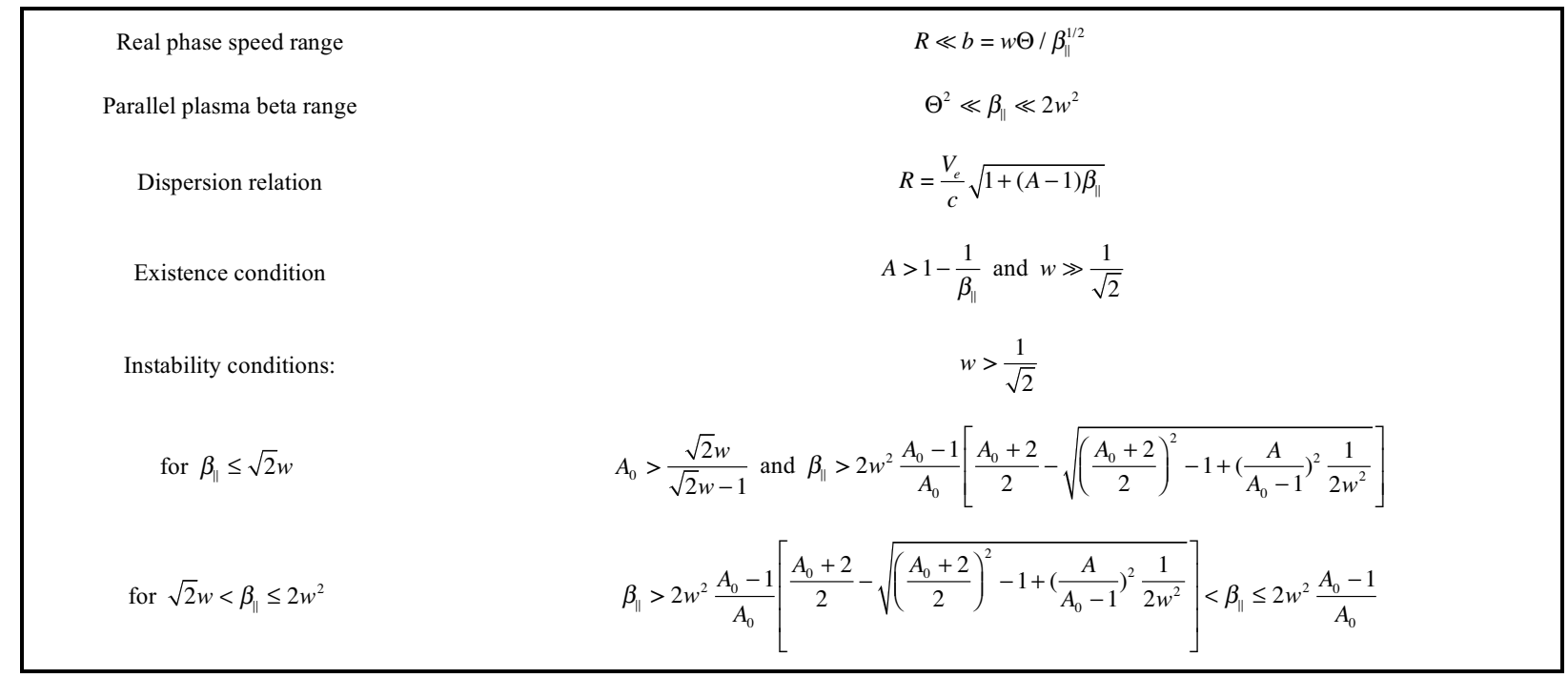

which for large values of $A_{0} \gg 1$ yields $A_{0}>\left(2 w^{2}-1\right) / \beta_{\|}$. For values of $A_{0}$ close to $\sqrt{2} w /(\sqrt{2} w-1)$ we approximate the right-hand side of constraint $((77))$ further to

$\beta_{\|}>\left(g^{2}-1\right) \frac{\left(A_{0}-\frac{g}{g-1}\right)}{2\left(A_{0}-1\right)}$

yielding

$A_{0}>\frac{g(g+1)-2 \beta_{\|}}{g^{2}-1-2 \beta_{\|}}$

which is also shown in Fig. (3). In Table 1 we summarize the properties of the unstable weakly amplified pair Alfven mode.

\subsection{Pair Cyclotron Waves}

Now dropping the assumption $R \ll b$ and scaling $R=b x$, we obtain for the dispersion relation (56) for equal positron and electron anisosotropies $\left(A_{p}=A_{e}=A_{0}\right)$

$$
\begin{aligned}
& 0=\Re \Lambda_{R H, L H}(x, S=0)=b^{2} x^{2}-1+\frac{2 w^{2} x^{2}}{1-x^{2}} \\
& +\beta_{\|}\left[\frac{1+3 x^{2}}{\left(1-x^{2}\right)^{3}}-\frac{A_{0}\left(1+x^{2}\right)}{\left(1-x^{2}\right)^{2}}\right]
\end{aligned}
$$

The conditions $X_{1,2} \gg 1$ demand that $\beta_{\|} \ll 2 w^{2}$ and

$$
x \leq 1-\frac{\beta_{\|}^{1 / 2}}{w}
$$

In a high-density plasma $b \ll w$ and with $x \ll 3$ the dispersion relation (80) simplifies to

$$
0=\Re \Lambda_{R H, L H}(x, S=0) \simeq-1+\frac{2 w^{2} x^{2}}{1-x^{2}}+\frac{\beta_{\|}}{\left(1-x^{2}\right)^{3}}\left[3 x^{2}+1-A_{0}\right]
$$

For small plasma beta $\beta_{\|}<1$ we obtain the solution

$$
x \simeq \frac{1}{\sqrt{1+2 w^{2}}}
$$

which for large $2 w^{2} \gg 1$ reduces to the four pair Alfven wave solutions $(60)$. In the opposite case $w \ll 2^{-1 / 2}$ the solution (83) yields the four pair cyclotron waves with the dispersion relation

$R=b x \simeq b\left[1-w^{2}\right]$

The constraint (81) demands $\beta_{\|} \leq w^{6}$ which is fulfilled for small values of $w$ because $\beta_{\|} \leq w^{2}$.

Eq. (82) for small plasma beta $\beta_{\|}<1$ also provides

$$
\frac{\partial \Re \Lambda_{R H, L H}(R, S=0)}{\partial R} \simeq \frac{4 w^{2} x}{b\left(1-x^{2}\right)^{2}}>0,
$$

so that with Eqs. (25) and (53) the growth/damping rate reads

$$
\begin{aligned}
& S_{R H, L H}\left(A_{p}=A_{e}=A_{0}\right)=\frac{\pi^{1 / 2}}{2} \frac{b^{2}}{\Theta} \frac{\left(1-x^{2}\right)^{2}}{x} e^{-\frac{b^{2}\left(1+x^{2}\right)}{\Theta^{2}}} \\
& \cosh \left(\frac{2 b^{2} x}{\Theta^{2}}\right)\left[\left(A_{0}-1\right) \tanh \left(\frac{2 b^{2} x}{\Theta^{2}}\right)-A_{0} x\right]
\end{aligned}
$$

For $A_{0} \leq 1$, including the case of isotropic distribution functions, this rate is always negative. In order to drive the pair cyclotron waves unstable possible values with $A_{0}>1$ are required such that

$$
\left(A_{0}-1\right) \tanh \left(\frac{2 b R}{\Theta^{2}}\right)>\frac{A_{0} R}{b}
$$

Approximating the tanh-function by Eq. (67) yields 


$$
\frac{A_{0}-1}{A_{0}}>\frac{\beta_{\|}}{2 w^{2}}+\frac{R}{b}=\frac{\beta_{\|}}{2 w^{2}}+1-w^{2}
$$

upon insertion of the solution (84) for $w \ll 2^{-1 / 2}$. We obtain

$$
A_{0}>\frac{1}{w^{2}\left(1-\left(\frac{\beta_{\|}}{2 w^{4}}\right)\right)}
$$

Only for large values of the anisotropy $\left(A_{0} \geq\left(2 w^{2}\right)^{-1} \gg 1\right)$ pair cyclotron waves can be excited at small plasma betas $\beta_{\|}<2 w^{4} \ll 1$ provided $w \ll 2^{-1 / 2}$. In Table 2 we summarize the properties of the unstable weakly amplified pair cyclotron wave mode.

Table 2. Properties of Weakly Amplified Pair Cyclotron Wave Mode

\begin{tabular}{|cc|}
\hline Real phase speed range & $R \ll b\left(1-\left(\beta_{\|}^{1 / 2} / w\right)\right)$ \\
Parallel plasma beta range & $\Theta^{2} \ll \beta_{\|} \ll 2 w^{2}$ \\
Dispersion relation & $R=b\left(1-w^{2}\right)$ \\
Existence condition & $w \ll \frac{1}{\sqrt{2}}$ \\
Instability conditions: & $w \ll \frac{1}{\sqrt{2}}$ \\
for $\beta_{\|} \leq 2 w^{4}$ & $A_{0}>\frac{1}{w^{2}\left(1-\left(\frac{\beta_{\|}}{2 w^{4}}\right)\right)}$ \\
\hline
\end{tabular}

\subsection{Region WII: Electromagnetic Light at Large} Frequencies $R \gg b+\Theta$

For large frequencies $R \gg b+\Theta$ the dispersion relation (56) reduces to

$$
\begin{aligned}
& 0=\Re \Lambda_{R H, L H}(R, S=0) \simeq R^{2}-\left(1+2 w^{2}\right) \\
& -2 w^{2} \frac{b^{2}}{R^{2}}\left[1+\frac{\Theta^{2}\left(A_{p}+A_{e}\right)}{4 b^{2}}\right]
\end{aligned}
$$

and

$$
0=\mathfrak{I} \Lambda_{R H, L H}(R, S=0) \simeq \pi^{1 / 2} w^{2} \frac{R}{\Theta}\left(A_{p}+A_{e}\right) e^{-R^{2} / \Theta^{2}},
$$

so that for both polarisations to lowest order in $(b / R)^{2}$ we obtain the dispersion relation of electromagnetic light

$$
R^{2} \simeq 1+2 w^{2}
$$

with the same damping rate

$$
S=-\pi^{1 / 2} w^{2} \frac{A}{\Theta} e^{-R^{2} / \Theta^{2}}
$$

\section{WEAKLY PROPAGATING SOLUTIONS}

For weakly propagating fluctuations with positive $S>0$ Eq. (34)-(37) read

$$
\begin{aligned}
& 0=\Re \Lambda_{R H}(R=0, S)=-S^{2}-1- \\
& \frac{w^{2}}{2}\left[4+A_{p} \Re Z^{\prime}\left(\frac{i S+b}{\Theta_{p}}\right)+A_{e} \Re Z^{\prime}\left(\frac{i S-b}{\Theta_{e}}\right)\right] \\
& +w^{2} b\left[\frac{1}{\Theta_{e}} \mathfrak{R} Z\left(\frac{i S-b}{\Theta_{e}}\right)-\frac{1}{\Theta_{p}} \mathfrak{\Re Z}\left(\frac{i S+b}{\Theta_{p}}\right)\right], \\
& 0=\mathfrak{I} \Lambda_{R H}(R=0, S)=-\frac{w^{2}}{2}\left[A_{p} \mathfrak{I} Z^{\prime}\left(\frac{i S+b}{\Theta_{p}}\right)+A_{e} \mathfrak{I} Z^{\prime}\left(\frac{i S-b}{\Theta_{e}}\right)\right] \\
& +w^{2} b\left[\frac{1}{\Theta_{e}} \mathfrak{I} Z\left(\frac{i S-b}{\Theta_{e}}\right)-\frac{1}{\Theta_{p}} \mathfrak{I} Z\left(\frac{i S+b}{\Theta_{p}}\right)\right],
\end{aligned}
$$

and

$$
\begin{aligned}
& 0=\Re \Lambda_{L H}(R=0, S)=-S^{2}-1- \\
& \frac{w^{2}}{2}\left[4+A_{p} \Re Z^{\prime}\left(\frac{i S-b}{\Theta_{p}}\right)+A_{e} \Re Z^{\prime}\left(\frac{i S+b}{\Theta_{e}}\right)\right] \\
& +w^{2} b\left[\frac{1}{\Theta_{p}} \Re Z\left(\frac{i S-b}{\Theta_{p}}\right)-\frac{1}{\Theta_{e}} \mathfrak{R Z}\left(\frac{i S+b}{\Theta_{e}}\right)\right], \\
& 0=\mathfrak{I} \Lambda_{L H}(R=0, S)=-\frac{w^{2}}{2}\left[A_{p} \mathfrak{I} Z^{\prime}\left(\frac{i S-b}{\Theta_{p}}\right)+A_{e} \mathfrak{I} Z^{\prime}\left(\frac{i S+b}{\Theta_{e}}\right)\right] \\
& +w^{2} b\left[\frac{1}{\Theta_{p}} \mathfrak{I Z}\left(\frac{i S-b}{\Theta_{p}}\right)-\frac{1}{\Theta_{e}} \mathfrak{I} Z\left(\frac{i S+b}{\Theta_{e}}\right)\right]
\end{aligned}
$$

The absolute value of the arguments of the plasma dispersion function for equal parallel plasma temperatures $\Theta_{e}=\Theta_{p}=\Theta$ is given by

$$
Y=\left|\frac{i S \pm b}{\Theta}\right|=\frac{\sqrt{S^{2}+b^{2}}}{\Theta}
$$

Expressed in terms of the plasma beta (43) the condition $Y=1$ corresponds to

$$
S 1\left(\beta_{\|}\right)=\Theta \sqrt{1-w^{2} \beta_{\|}^{-1}}
$$

in the case of a finite magnetic field $(b \neq 0)$, whereas for unmagnetized plasmas $S 1(b=0)=\Theta$.

As illustrated in Fig. (4) the curve $S 1\left(\beta_{\|}\right)$for finite magnetic field strengths divides the $\left(S, \beta_{\|}\right)$-solution plane into the two regions SI, where $Y \gg 1$, and SII, where $Y \ll 1$. For orientation we also plot the $b\left(\beta_{\|}\right)$-dependence (43) that indicates the location of the pair cyclotron phase speed $b$ in this diagram. In the unmagnetized $(b=0)$ case, the region SI with $Y \gg 1$ corresponds to values of $S \gg \Theta$, whereas region SII with $Y \ll 1$ corresponds to values of $S \ll \Theta$. Viewed as a function of the parallel plasma beta, the region SI applies to all plasma beta values provided $S>\Theta$, whereas it is limited to values $\beta_{\|}<w^{2}$ for $S<\Theta$. 


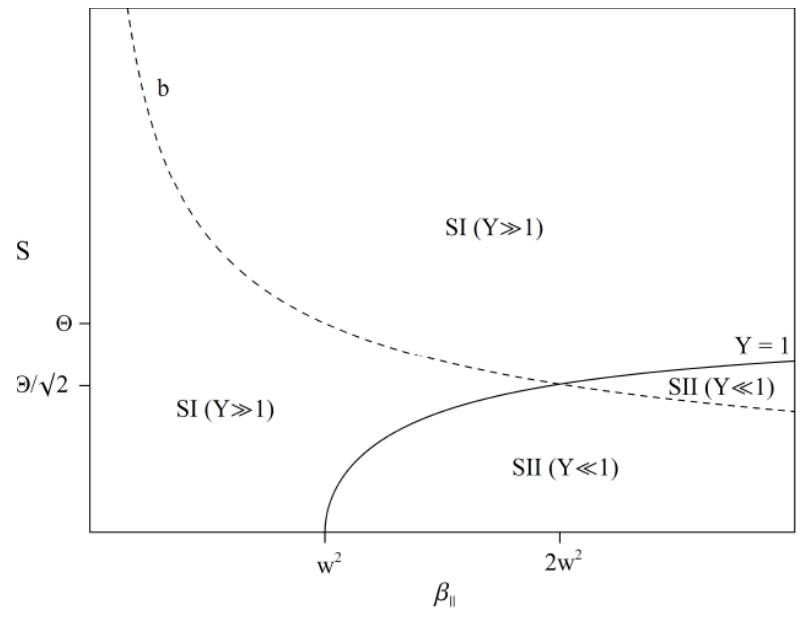

Fig. (4). Separation of the $S-\beta_{\|}$-parameter plane into two different regions depending on the values of $Y(S)$ being small or large compared to unity. $S$ is the argument of the plasma dispersion function in the weak propagation limit. For orientation the dashed curve shows the $b\left(\beta_{\|}\right)$-dependence (43) that indicates the location of the pair cyclotron phase speed $b$ in this diagram.

In the large argument case $Y \gg 1$ for region SI we use expansion (7) for growing solutions $S>0$, implying $\sigma=0$, to obtain to second order in $\Theta \ll 1$

$$
\begin{aligned}
& Z\left(\frac{i S \pm b}{\Theta}\right) \simeq \frac{\mp b \Theta^{2}}{b^{2}+S}\left[1+\frac{\Theta^{2}\left(b^{2}-3 S^{2}\right)}{2\left(b^{2}+S^{2}\right)^{2}}\right] \\
& +i \frac{\Theta S}{b^{2}+S^{2}}\left[1+\frac{\Theta^{2}\left(3 b^{2}-S^{2}\right)}{2\left(b^{2}+S^{2}\right)^{2}}\right]
\end{aligned}
$$

and

$Z^{\prime}\left(\frac{i S \pm b}{\Theta}\right) \simeq\left(\frac{\Theta^{2} b^{2}-S^{2}}{\left(b^{2}+S^{2}\right)^{2}}\right) \mp i \frac{\Theta^{2} b S}{2\left(b^{2}+S^{2}\right)^{2}}$

Likewise, in the small argument case $Y \ll 1$ appropriate for region SII we use expansion ((6)) to obtain

$$
\begin{aligned}
& Z\left(\frac{i S \pm b}{\Theta}\right) \simeq \mp \frac{2 b}{\Theta}\left[1-\frac{2\left(b^{2}-3 S^{2}\right)}{3 \Theta^{2}}\right] \pm \pi^{1 / 2} \sin \left(\frac{2 b S}{\Theta^{2}}\right) e^{\frac{S^{2}-b^{2}}{\Theta^{2}}} \\
& +i\left(\pi^{1 / 2} \cos \left(\frac{2 b S}{\Theta^{2}}\right) e^{+\frac{S^{2}-b^{2}}{\Theta^{2}}}-\frac{2 S}{\Theta}\left[1+\frac{2\left(S^{2}-3 b^{2}\right)}{3 \Theta^{2}}\right]\right)
\end{aligned}
$$

and

$$
\begin{aligned}
& Z^{\prime}\left(\frac{i S \pm b}{\Theta}\right) \simeq-2\left[1+\frac{2\left(S^{2}-b^{2}\right)}{\Theta^{2}}\right]+ \\
& \frac{2 \pi^{1 / 2}}{\Theta} e^{+\frac{S^{2}-b^{2}}{\Theta^{2}}}\left[S \cos \left(\frac{2 b S}{\Theta^{2}}\right)+b \sin \left(\frac{2 b S}{\Theta^{2}}\right)\right] \\
& \pm i\left(\frac{8 b S}{\Theta^{2}}-\frac{2 \pi^{1 / 2}}{\Theta} e^{+\frac{S^{2}-b^{2}}{\Theta^{2}}}\left[S \sin \left(\frac{2 b S}{\Theta^{2}}\right)+b \cos \left(\frac{2 b S}{\Theta^{2}}\right)\right]\right)
\end{aligned}
$$

\subsection{The Weibel Instability in the Unmagnetized Case} $b=0$

It is instructive to start with the unmagnetized case $b=0$ considered first by Weibel [24].

\subsubsection{Hot Weibel Mode in Region SI with $S \gg \Theta$}

Here the expansions (100) and (101) apply yielding with $b=0$ that

$Z\left(\frac{i S}{\Theta}\right) \simeq i \frac{\Theta}{S}\left[1-\frac{\Theta^{2}}{2 S^{2}}\right]$

is purely imaginary, whereas

$Z^{\prime}\left(\frac{i S}{\Theta}\right) \simeq-\frac{\Theta^{2}}{S^{2}}$

is purely real, i.e. $\mathfrak{I} Z\left(\frac{i S}{\Theta}\right)=0$. According to Eqs. (32), (95) and (97) any growing solution then is aperiodic with $R=0$. The RH and LH polarized dispersion relations (94) and (96) in this case agree and are

$\Re \Lambda_{R H, L H}(R=0, S)=-S^{2}-\left(1+2 w^{2}\right)+\frac{A \Theta^{2} w^{2}}{S^{2}}=0$,

where we use the combined plasma temperature anisotropy (59). Because $\Theta \ll 1$ the high-temperature hot Weibel solution of Eq. (106) is

$S^{2}=\frac{1+2 w^{2}}{2}\left[\sqrt{1+\frac{4 A \Theta^{2} w^{2}}{\left(1+2 w^{2}\right)^{2}}}-1\right] \simeq \frac{A \Theta^{2} w^{2}}{1+2 w^{2}}$,

subject to the condition $S^{2} \gg \Theta^{2}$ which requires that the combined anisotropy

$A \gg \frac{1+2 w^{2}}{w^{2}}=2+\frac{1}{w^{2}}$

is much larger than 2 at small wavenumber values. For vanishing temperature anisotropies $A_{p}=A_{e}=A=1$ the condition (108) cannot be fulfilled. The corresponding growth rate for large enough $A$

$\gamma^{2}=S^{2} k^{2} c^{2}=\frac{w_{p, e}^{2} A \Theta^{2} k^{2} c^{2}}{2\left(k^{2} c^{2}+2 w_{p}^{2}, e\right)}$

increases linearly $(\gamma \propto|k|)$ at small wavenumber values. For given $A$ the condition (108) cannot be fulfilled at

$w^{-2}=\frac{k^{2} c^{2}}{\omega_{p, e}^{2}}>(A-2)$,

so that the maximum growth rate is

$\gamma_{\max }=\sqrt{A-2} \Theta \omega_{p, e}$

\subsubsection{Cool Weibel Mode in Region SII with $S \ll \Theta$} that

The small argument asymptotics (103) yields for $b=0$ 
$Z^{\prime}\left(\frac{i S}{\Theta}\right) \simeq 2 \pi^{1 / 2} \frac{S}{\Theta} e^{\frac{S^{2}}{\Theta^{2}}}-2-4 \frac{S^{2}}{\Theta^{2}} \simeq-2+2 \pi^{1 / 2} \frac{S}{\Theta}$

is purely real, i.e. $\mathfrak{I} Z\left(\frac{i S}{\Theta}\right)=0$. According to Eq. (32) any growing solution then is aperiodic with $R=0$. The RH and LH polarized dispersion relations (94) and (96) in this case agree and lead to

$$
S^{2}+1+2(1-A) w^{2}+2 \pi^{1 / 2} w^{2} A \frac{S}{\Theta}=0
$$

No real solutions for $S$ exist for $A \leq 1$, including the isotropic plasma case. For anisotropies $A>1$ we find the cool-Weibel solution

$S \simeq \Theta \frac{2(A-1) w^{2}-1}{2 \pi^{1 / 2} A w^{2}}$

if $2(A-1) w^{2}>1$, which requires that

$$
A>1+\frac{1}{2 w^{2}}=\frac{1+2 w^{2}}{2 w^{2}}
$$

which is weaker than condition (108). Apparently, in an unmagnetized plasma the low-temperature Weibel solution (114) can be realized under the condition (115).

\subsection{Region SI with $Y \gg S 1$ for Finite Magnetic Fields} $b \neq 0$

With the large argument asymptotics (100) and (101) we obtain for the dispersion relations (94)-(97)

$$
\begin{aligned}
& 0=\Re \Lambda_{R H}(R=0, S)=\Re \Lambda_{L H}(R=0, S)=-S^{2}-1-2 w^{2} \\
& +w^{2}\left(\frac{2 b^{2}}{b^{2}+S^{2}}-\frac{\Theta^{2}\left(A_{p}+A_{e}\right)\left(b^{2}-S^{2}\right)}{2\left(b^{2}+S^{2}\right)^{2}}+\frac{\Theta^{2} b^{2}\left(b^{2}-3 S^{2}\right)}{\left(S^{2}+b^{2}\right)^{3}}\right) \\
& =-\left[S^{2}+1+2 w^{2} \frac{S^{2}}{b^{2}+S^{2}}\right]+w^{2} \Theta^{2}\left[\frac{A\left(S^{4}-b^{4}\right)+b^{4}-3 b^{2} S^{2}}{\left(S^{2}+b^{2}\right)^{3}}\right]
\end{aligned}
$$

and

$$
0=\mathfrak{I} \Lambda_{R H}(R=0, S)=-\mathfrak{S} \Lambda_{L H}(R=0, S)=\frac{w^{2} \Theta^{2}\left(A_{p}-A_{e}\right) b S}{\left(b^{2}+S^{2}\right)^{2}}
$$

We note that only for different positron and electron temperature anisotropies $\left(A_{p} \neq A_{e}\right)$ the imaginary part (117) is non-vanishing. According to Eq. (32) in a magnetized plasma we therefore obtain non-zero real parts for $A_{p} \neq A_{e}$. For equal $\left(A_{p}=A_{e}\right.$ ) anisotropies the weakly propagating fluctuations in a magnetized plasma will be purely aperiodic.

Scaling

$$
S^{2}=\Theta^{2} y, \quad b^{2}=\alpha \Theta^{2}, \alpha=w^{2} / \beta_{\|}
$$

the real part (116) becomes

$$
\begin{aligned}
0=\mathfrak{R} \Lambda_{R H, L H}(y)= & -\left[\Theta^{2} y+1+2 w^{2} \frac{y}{\alpha+y}\right] \\
& +w^{2}\left[\frac{A\left(y^{2}-\alpha^{2}\right)-3 \alpha y+\alpha^{2}}{(\alpha+y)^{3}}\right]
\end{aligned}
$$

which for $S \simeq \Theta$ holds for all $y$, whereas for $S \ll \Theta$ it holds for plasma betas $\beta_{\|} \ll w^{2}$ (see Fig. 4), corresponding to $\alpha \gg 1$. Because $\Theta^{-2} \gg 1$ Eq. (119) for $y \leq \Theta^{-2}$ is independent of the plasma temperature reading

$$
\begin{aligned}
0= & \Re \Lambda_{R H, L H}\left(y \leq \Theta^{-2}\right) \simeq-\left(1+2 w^{2} \frac{y}{y+\alpha}\right) \\
& +w^{2}\left[\frac{2 \alpha(\alpha+y)^{2}+A\left(y^{2}-\alpha^{2}\right)+\alpha(\alpha-3 y)}{(\alpha+y)^{3}}\right]
\end{aligned}
$$

Now we scale $y=\alpha t$, which implies $S^{2}=b^{2} t$, so that the dispersion relations (117) and (119) read

$$
0=\mathfrak{I} \Lambda_{R H, L H}(t)= \pm \beta_{\|}\left(A_{p}-A_{e}\right) \frac{t^{1 / 2}}{(1+t)^{2}}
$$

and

$$
\begin{aligned}
& 0=\Re \Lambda_{R H, L H}(t)=-\left[1+\frac{2 w^{2} t}{1+t}\right]+\beta_{\|} H(t, A), \\
& H(t, A)=\frac{A\left(t^{2}-1\right)-3 t+1}{(t+1)^{3}}
\end{aligned}
$$

Eq. (122) provides the relation between the combined temperature anisotropy $A$ and the parallel plasma beta to drive weakly propagating fluctuations in magnetized plasmas in region SI. Evidently, it is a polynomial of fourth order in the variable $t$ which in the following we will simplify to quadratic polynomials.

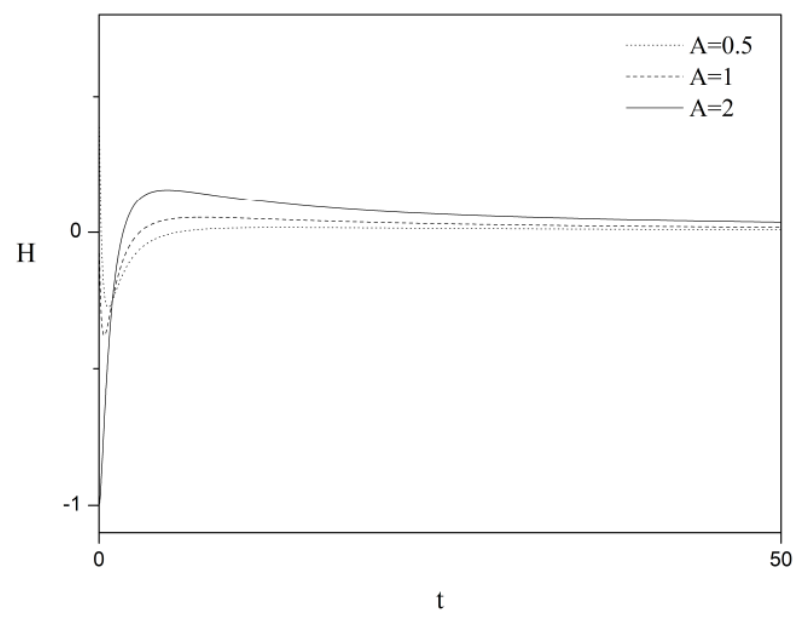

Fig. (5). The function $H(t, A)$ for the three values $A=0.5,1,2$ for large values of $t$. 
The function $H(t, A)$ is sketched in Figs. $(\mathbf{5}, \mathbf{6})$ for large and small values of $t$ for the three values $A=0.5,1,2$. The function $H$ has two zeros at

$t_{1}=\frac{1}{2 A}[3+\sqrt{4 A(A-1)+9}] \geq 1$,

$t_{2}=\frac{1}{2 A}[3+\sqrt{4 A(A-1)+9}] \leq(1-A)$

For anisotropies $A \geq 1$ not smaller than unity the function $H$ starts from a negative value at $t=0$, is negative for $0<t<t_{1}$ and exhibits one maximum at values larger than $t_{1}$. In this case, for large enough parallel plasma beta $\beta_{\|}$, the dispersion relation (122) then has two solutions at large values of $t>t_{1}$ which we will later identify as mirror and pair cyctronic fluctuations.

For small anisotropies $A<1$ (see Fig. 5 for $A=0.5$ ), a second maximum of the function $H$ exists at $t=0$ with $H_{\max }=1-A$. In this case a third solution to Eq. (122) close to $t=0$ exists for large enough $\beta_{\|}$which we will later identify as firehose fluctuations.

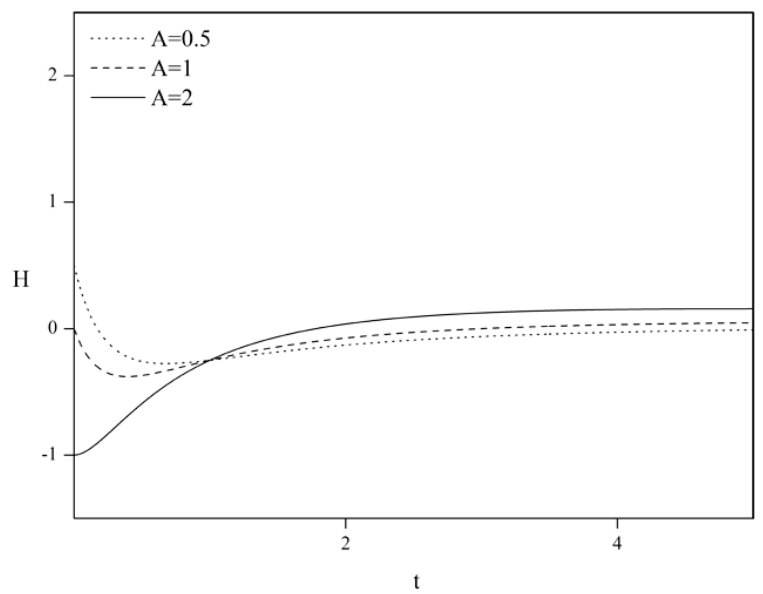

Fig. (6). The function $H(t, A)$ for the three values $A=0.5,1,2$ for small values of $t$.

For the following analysis it is convenient to substitute

$t=\delta+(\delta+1) x$,

with

$\delta=\frac{3}{2 A} \in[0, \infty]$

The dispersion relation (122) then leads to the equation

$L(x, \delta)=f(x, \delta)$

with the functions

$f(x, \delta)=\frac{3 \beta_{\|}}{2 \delta(\delta+1)} h(x, \delta), \quad h(x, \delta)=\frac{x^{2}-K^{2}(\delta)}{(x+1)^{3}}$,

where
$K^{2}(\delta)=1-\frac{8 \delta}{3(\delta+1)^{2}}>0$,

and

$L(x, \delta)=1+2 w^{2} \frac{x+\frac{\delta}{\delta+1}}{x+1}$

which is slowly varying with $x$. For small and large values of $\delta$ the value of $K^{2}$ is close to unity. For $\delta=1$ the minimum value $K_{\min }^{2}=1 / 3$ occurs.

\subsubsection{The Anisotropy Function $h(x, \delta)$}

The first and second derivatives of the anisotropy function (127) are given by

$\frac{d h}{d x}=\frac{-x^{2}+2 x+3 K^{2}}{(1+x)^{4}}$

and

$\frac{d^{2} h}{d x^{2}}=\frac{2}{(x+1)^{5}}\left[x^{2}-4 x+1-6 K^{2}\right]$

yielding a maximum at

$x_{1}=1+\sqrt{1+3 K^{2}}>1+\sqrt{2}=2.414$

and a minimum at

$x_{2}=1-\sqrt{1+3 K^{2}}<0$

For all anisotropies $\delta$ the minimum value $-0.414 \leq x_{2} \leq 0$ is negative. The maximum value $x_{1}$ has its smallest value 2.414 at $\delta=1$.

For the maximum value of the function $h$ at positive values of $x$ we find

$h_{\max }(\delta)=h\left(x_{1}, \delta\right)=\frac{2\left(1+K^{2}+\sqrt{1+3 K^{2}}\right)}{\left(2+\sqrt{1+3 K^{2}}\right)^{3}} \simeq \frac{1}{8}$

because $K^{2} \simeq 1$ for small and large $\delta$. The maximum value of the anisotropy function $f$ then is

$f_{\max } \simeq \frac{3 \beta_{\|}}{16 \delta(\delta+1)}$

\subsubsection{Instability Condition for Solutions at $x>K$}

According to Eq. (126) solutions can be found if

$f_{\max }(\delta) \geq L\left(x_{1}, \delta\right)$,

yielding to leading order

$\frac{3 \beta_{\|}}{16 \delta(1+\delta)} \geq 1+2 w^{2}$

where we use again $K^{2} \simeq 1$. We obtain 
$\delta \leq \frac{1}{2}\left[\sqrt{1+\frac{3 \beta_{\|}}{4\left(1+2 w^{2}\right)}}-1\right]$

Eq. (141) implies the instability condition

$$
A \geq \frac{3}{\sqrt{1+\frac{3 \beta_{\|}}{4\left(1+2 w^{2}\right)}-1}},
$$

with the asymptotics for small and large values of the parallel plasma beta

$A\left(\beta_{\|} \ll \frac{4}{3}\left(1+2 w^{2}\right)\right) \geq \frac{8\left(1+2 w^{2}\right)}{\beta_{\|}}$

and

$A\left(\beta_{\|} \gg \frac{4}{3}\left(1+2 w^{2}\right)\right) \geq \sqrt{\frac{12\left(1+2 w^{2}\right)}{\beta_{\|}}}$

\subsubsection{Approximate Solutions at Positive $x$}

Here we derive approximated solutions to Eq. (126) at positive $x$ by assuming again $K(\delta)=1$ which is well fulfilled for small and large values of $\delta$. The function $f$ then reduces to

$f(x, \delta \neq 1)=\frac{3 \beta_{\|}}{2 \delta(\delta+1)} \frac{x-1}{(x+1)^{2}}$,

whereas the function (129) approaches the constant $L(x \gg K) \simeq 1+2 w^{2}$. We find the two solutions

$x_{1,2}=D-1 \pm \sqrt{D(D-4)}$

with

$$
D=\frac{3 \beta_{\|}}{4 \delta(\delta+1)\left(1+2 w^{2}\right)}
$$

The solutions (143) are real provided that $D \geq 4$, which corresponds exactly to the instability condition (137).

According to the substitution (124) the two solutions (143) imply with $S^{2}=b^{2} t=b^{2}[\delta+(\delta+1) x]$ as solutions

$$
\begin{aligned}
& S_{1}^{2}=\frac{3 \beta_{\|} b^{2}}{4 \delta\left(1+2 w^{2}\right)}\left[1+\sqrt{1-\frac{16 \delta(\delta+1)\left(1+2 w^{2}\right)}{3 \beta_{\|}}}\right]-b^{2} \\
& =\frac{3 \Theta^{2} w^{2}}{4 \delta\left(1+2 w^{2}\right)}\left[1+\sqrt{1-\frac{16 \delta(\delta+1)\left(1+2 w^{2}\right)}{3 \beta_{\|}}}\right]-b^{2}
\end{aligned}
$$

and

$$
\begin{aligned}
& S_{2}^{2}=\frac{3 \beta_{\|} b^{2}}{4 \delta\left(1+2 w^{2}\right)}\left[1-\sqrt{1-\frac{16 \delta(\delta+1)\left(1+2 w^{2}\right)}{3 \beta_{\|}}}\right]-b^{2} \\
& =\frac{3 \Theta^{2} w^{2}}{4 \delta\left(1+2 w^{2}\right)}\left[1-\sqrt{1-\frac{16 \delta(\delta+1)\left(1+2 w^{2}\right)}{3 \beta_{\|}}}\right]-b^{2}
\end{aligned}
$$

which for $S>\Theta$ hold for all values of $\beta_{\|}$, whereas for $S<\Theta$ only for $\beta_{\|}<w^{2}$.

The instability condition in the form (137) allows the approximations

$$
\begin{aligned}
& S_{1}^{2} \simeq \frac{3 \beta_{\|} b^{2}}{2 \delta\left(1+2 w^{2}\right)}-b^{2}(2 \delta+3)=\frac{A \Theta^{2} w^{2}}{\left(1+2 w^{2}\right)}-3 b^{2}\left(\frac{1}{A}+1\right) \\
& =\Theta^{2} w^{2}\left[\frac{A}{1+2 w^{2}}-\frac{3(A+1)}{A \beta_{\|}}\right]
\end{aligned}
$$

and

$$
S_{2}^{2} \simeq(1+2 \delta) b^{2}=\left(\frac{3}{A}+1\right) b^{2}=\frac{\Theta^{2} w^{2}}{\beta_{\|}}\left(\frac{3}{A}+1\right)
$$

\subsubsection{Mirror Fluctuations}

We first investigate the limit of a vanishing magnetic field $b \rightarrow 0$ and $\beta_{\|} \rightarrow \infty$. Solution $S_{2}^{2}=0$ vanishes whereas

$S_{1}^{2}(b=0)=\frac{A \Theta^{2} w^{2}}{\left(1+2 w^{2}\right)}$

agrees exactly with the earlier derived hot-Weibel solution (107). This remarkable consistency justifies the approximations made in this subsection. Solution (147) evidently is the modification of the Weibel instability for finite magnetic fields sometimes referred to as the magnetic Weibel instability [25], although the classification as mirror instability is more appropriate. In order to exist, besides obeying the instability condition (139), it has to be positive which sets an upper limit on the magnetic field strength

$b^{2}<\frac{A^{2}}{A+1} \frac{\Theta^{2} w^{2}}{3\left(1+2 w^{2}\right)}$

This upper limit on $b$ corresponds to a lower limit on the plasma beta

$\beta_{\|}>\frac{3(A+1)}{A^{2}}\left(1+2 w^{2}\right)$,

which is equivalent to

$A>\frac{3\left(1+2 w^{2}\right)}{2 \beta_{\|}}\left[1+\sqrt{1+\frac{4 \beta_{\|}}{3\left(1+2 w^{2}\right)}}\right]$

For $\beta_{\|}<1+2 w^{2}$ this yields

$A\left(\beta_{\|}<1+2 w^{2}\right)>\frac{3\left(1+2 w^{2}\right)}{\beta_{\|}}$,

in good agreement with the earlier condition (140). Alternatively, for $\beta_{\|} \gg 1+2 w^{2}$ we obtain in agreement with the earlier condition (141)

$A\left(\beta_{\|} \gg 1+2 w^{2}\right)>\sqrt{\frac{3\left(1+2 w^{2}\right)}{\beta_{\|}}}$ 
Table 3. Properties of Weakly Propagating Mirror Fluctuations

\begin{tabular}{|c|c|}
\hline Imaginary phase speed range & $S>0$ for $\Theta^{2}<\beta_{\|}<w^{2}$ and $S>\Theta \sqrt{1-\frac{w^{2}}{\beta_{\|}}}$for $\beta_{\|} \geq w^{2}$ \\
\hline Real phase speed & aperiodic $R=0$ for $A_{p}=A_{e}$ \\
\hline Parallel plasma beta range & all $\beta_{\|}>\Theta^{2}$ \\
\hline Dispersion relation & $S_{1} \simeq \Theta w\left[\frac{A}{1+2 w^{2}}-\frac{3(A+1)}{A \beta_{\|}}\right]^{1 / 2}$ \\
\hline Existence condition & $A>2+w^{-2}$ for $\beta_{\|}>1+2 w^{2}$ \\
\hline Instability conditions: & $A \geq \frac{3}{\sqrt{1+\frac{3 \beta_{\|}}{4\left(1+2 w^{2}\right)}}-1}$ \\
\hline Limit in unmagnetized plasmas $\left(b=0, \beta_{\|}=\infty\right)$ & hot-Weibel mode \\
\hline
\end{tabular}

To be used for large values of $\beta_{\|}>w^{2}$, the solution $S_{1}$ also has to fulfil $S_{1}^{2}>\Theta^{2}$ yielding the additional condition

$A>\frac{1+2 w^{2}}{2 w^{2}} \frac{w^{2}+\beta_{\|}}{\beta_{\|}}\left[1+\sqrt{1+\frac{12 \beta_{\|} w^{2}}{\left(1+2 w^{2}\right)^{2}\left(w^{2}+\beta_{\|}\right)^{2}}}\right]$

The properties of the weakly propagating mirror fluctuations are summarized in Table 3 .

\subsubsection{Pair Cyctronic Fluctuations}

The newly found weakly propagating solution $S_{2}$ will be referred to as pair cyctronic fluctuations, because the values of $S_{2}$ are close to the cyclotron phase speed. This is best seen when we insert the instability condition (139) in Eq. (148) yielding

$S_{2}^{2} \leq \sqrt{1+\frac{3 \beta_{\|}}{4\left(1+2 w^{2}\right)}} b^{2}$

which for region SI, where $\beta_{\|}<w^{2}$, is close to $S_{2}^{2}=b^{2}$.
For large $\beta_{\|} \gg 1+2 w^{2}$ the upper limit (156) can also be written as

$S_{2}^{2} \leq \sqrt{\frac{3}{4\left(1+2 w^{2}\right)}} \beta_{\|}^{1 / 2} b^{2}=\sqrt{\frac{3 w^{2}}{4\left(1+2 w^{2}\right)}} \Theta^{2} \frac{w}{\beta_{\|}^{1 / 2}} \ll \Theta^{2}$,

demonstrating that the pair cyclotron solution $S_{2}$ does not exist at large plasma betas $\beta_{\|} \gg w^{2}$ The properties of the weakly propagating pair cyctronic fluctuations are summarized in Table 4.

4.2.6. The Third Solution Near $t=0$ for Anisotropies $\delta>3 / 2$ : Firehose Fluctuations

We have remarked before that for anisotropies $A_{0}<1$, a third solution exists close $t=0$. This solution is identified as firehose fluctuation. Near $t=0$ we approximate Eq. (122) as

$0=\Re \Lambda_{R H, L H}(t) \simeq-\left[1+2 w^{2} t\right]+\beta_{\|}[(1-A)-3(2-A) t]$,

yielding the solution

Table 4. Properties of Weakly Propagating Pair Cyctronic Fluctuations

\begin{tabular}{|lc|}
\hline $\begin{array}{l}\text { Imaginary phase speed range } \\
\text { Real phase speed }\end{array}$ & $S>0$ for $\Theta^{2}<\beta_{\|}<w^{2}$ and $S>\Theta \sqrt{1-\frac{w^{2}}{\beta_{\|}}}$for $\beta_{\|}>w^{2}$ \\
$\begin{array}{c}\text { Parallel plasma beta range } \\
\text { Dispersion relation }\end{array}$ & aperiodic $R=0$ for $A_{p}=A_{e}$ \\
$\Theta^{2}<\beta_{\|}<w^{2}$ \\
Existence condition & $S_{2} \simeq b \sqrt{\frac{3+A}{A}}=\frac{\Theta w}{\beta_{\|}^{1 / 2}} \sqrt{\frac{3+A}{A}}$ \\
Instability conditions: & $A \geq \frac{3}{\sqrt{1+\frac{3 \beta_{\|}}{4\left(1+2 w^{2}\right)}}-1} \simeq \frac{8\left(1+2 w^{2}\right)}{\beta_{\|}}$ \\
& \\
Limit in unmagnetized plasmas $\left(b=0, \beta_{\|}=\infty\right)$ & not existing \\
\hline
\end{tabular}


Table 5. Properties of Weakly Propagating Firehose Fluctuations

\begin{tabular}{|cc|}
\hline $\begin{array}{c}\text { Imaginary phase speed range } \\
\text { Real phase speed }\end{array}$ & $S>0$ for $\Theta^{2}<\beta_{\|}<w^{2}$ \\
Parallel plasma beta range & aperiodic $R=0$ for $A_{p}=A_{e}$ \\
Dispersion relation & $1<\beta_{\|}<w^{2}$ \\
Existence condition & $S_{3} \simeq \Theta w\left[\frac{(1-A)-\beta_{\|}^{-1}}{2 w^{2}+3 \beta_{\|}(2-A)}\right]^{1 / 2}$ \\
Instability conditions: & $w^{2} \gg 1$ and $1<\beta_{\|}<w^{2}$ \\
& $A<1-\frac{1}{\beta_{\|}}$ \\
Limit in unmagnetized plasmas $\left(b=0, \beta_{\|}=\infty\right)$ & not existing \\
\hline
\end{tabular}

$t=\frac{\beta_{\|}(1-A)-1}{2 w^{2}+3 \beta_{\|}(2-A)}$

or

$S_{3}^{2}=b^{2} t=b^{2} \frac{\beta_{\|}(1-A)-1}{2 w^{2}+3 \beta_{\|}(2-A)}=\Theta^{2} w^{2} \frac{(1-A)-\beta_{\|}^{-1}}{2 w^{2}+3 \beta_{\|}(2-A)}$

This solution can only exist for parallel plasma betas with $\beta_{\|}(1-A)>1$ or

$A<1-\frac{1}{\beta_{\|}}$

which is only possible for high plasma betas $\beta_{\|}>1$. For $\beta_{\|}>w^{2}$ the solution $S_{3}$ does not exist because the requirement $S_{3}^{2}>\Theta^{2}$ at $\beta_{\|}>1$ needs

$A>\frac{6 \beta_{\|}+w^{2}}{2 \beta_{\|}-w^{2}}>2$,

in contradiction to condition (161). These dual conditions then require that $w^{2} \gg 1$, so that the solution $S_{3}$ then exists for $1<\beta_{\|}<w^{2}$. The properties of the weakly propagating firehose fluctuations are summarized in Table $\mathbf{5}$.

\subsection{Region SII with $Y \ll S 1$ for Finite Magnetic Fields $b \neq 0$}

The last case to be considered is region SII for non-zero magnetic fields $b \neq 0$ existing for $S \ll \Theta$ and $\beta_{\|}>2 w^{2}$ (see Fig. 4). The expansions (102) and (103) apply here, yielding for the dispersion relations (94)-(97)

$$
\begin{aligned}
& 0=\Re \Lambda_{R H, L H}(R=0, S)=-S^{2}-1-2(1-A)\left(1-\frac{2 w^{2}}{\beta_{\|}}\right) w^{2} \\
& -2 \pi^{1 / 2} w^{2} \frac{S}{\Theta}\left[A+(A-2) \frac{w^{2}}{\beta_{\|}}\right],
\end{aligned}
$$

and

$$
\begin{aligned}
& 0=\mathfrak{I} \Lambda_{R H, L H}(R=0, S)= \\
& -\frac{w^{2}}{2}\left(A_{p, e}-A_{e, p}\right)\left[\frac{8 b S^{2}}{\Theta}-\frac{2 \pi^{1 / 2}}{\Theta} e^{\frac{b^{2}-S^{2}}{\theta^{2}}}\left(S \sin \left(\frac{2 b S}{\Theta^{2}}\right)+b \cos \left(\frac{2 b S}{\Theta^{2}}\right)\right)\right] \\
& \simeq \frac{b w^{2}}{\Theta}\left(A_{p, e}-A_{e, p}\right)\left[\pi^{1 / 2}-\frac{4 S}{\Theta}\right]
\end{aligned}
$$

For $\beta_{\|} \rightarrow \infty$ the dispersion relation (163) reduces to the unmagnetized cool-Weibel dispersion relation (113). Eq. (163) also indicates that no real solutions for $S$ exist for $A \leq 1$, including the isotropic plasma case.

For anisotropies $A>1$ we find the low-temperature solution

$S_{4} \simeq \Theta \frac{2 w^{2}(A-1)\left(\beta_{\|}-w^{2}\right)-\beta_{\|}}{2 \pi^{1 / 2} w^{2}\left[A \beta_{\|}+(A-2) w^{2}\right]}$

provided that

$A>1+\frac{\beta_{\|}}{2 w^{2}\left(\beta_{\|}-2 w^{2}\right)}$

which only slightly modifies the unmagnetized condition (115) because $\beta_{\|}>2 w^{2}$ in region SII. In the case $w^{2}>1$ this modified low-temperature magnetic Weibel solution is a high-beta instability. In the case $w^{2} \ll 1$ it needs very large anisotropy values $A \propto\left(2 w^{2}\right)^{-1}$ to be excited which are much larger than the instability threshold anisotropies needed for the high-temperature mirror, firehose and pair cyctronic instabilities.

The solution $S_{4}$ is aperiodic for equal positron and electron $\left(A_{p}=A_{e}\right)$ anisotropies. The properties of the weakly propagating cool magnetized Weibel fluctuations are summarized in Table 6.

\section{RESULTS}

\subsection{Dispersion Relations}

In Fig. (7) we illustrate the dispersion relations of the weakly amplified pair Alfven waves $R / \Theta$ and the weakly 
propagating mirror, CMW (cold magnetized Weibel), firehose and cyctronic fluctuations $S / \Theta$ as a function of $x=\beta_{\|} / w^{2}$ in a bi-Maxwellian thermal pair plasma. We adopt a value of $w=10$ and anisotropies $A=5$ for the Alfven, mirror, cyclotron and firehose fluctuations and $A=0.2$ for the CMW-fluctuations. Note that for large values of $w$ the weakly amplified pair cyclotron waves do not exist. As illustrated, not all dispersion relations exist at all plasma beta values. In the limit of an unmagnetized plasma $\left(\beta_{\|} \propto x \rightarrow \infty\right)$ the mirror- and CMW-fluctuations approach the dispersion relations of the hot and cool Weibel fluctuations.

Table 6. Properties of Weakly Propagating Cool Magnetized Weibel Fluctuations

\begin{tabular}{|cc|}
\hline Imaginary phase speed range & $S<\Theta$ \\
Real phase speed & aperiodic $R=0$ for $A_{p}=A_{e}$ \\
Parallel plasma beta range & $\beta_{\|}>2 w^{2}$ \\
Dispersion relation & $S_{4} \simeq \Theta \frac{2 w^{2}(A-1)\left(\beta_{\|}-w^{2}\right)-\beta_{\|}}{2 \pi^{1 / 2} w^{2}\left[A \beta_{\|}+(A-2) w^{2}\right]}$ \\
Existence condition & $A>1$ \\
Instability conditions: & $A>1+\frac{\beta_{\|}}{2 w^{2}\left(\beta_{\|}-2 w^{2}\right)}$ \\
Limit in unmagnetized \\
plasmas $\left(b=0, \beta_{\|}=\infty\right)$
\end{tabular}

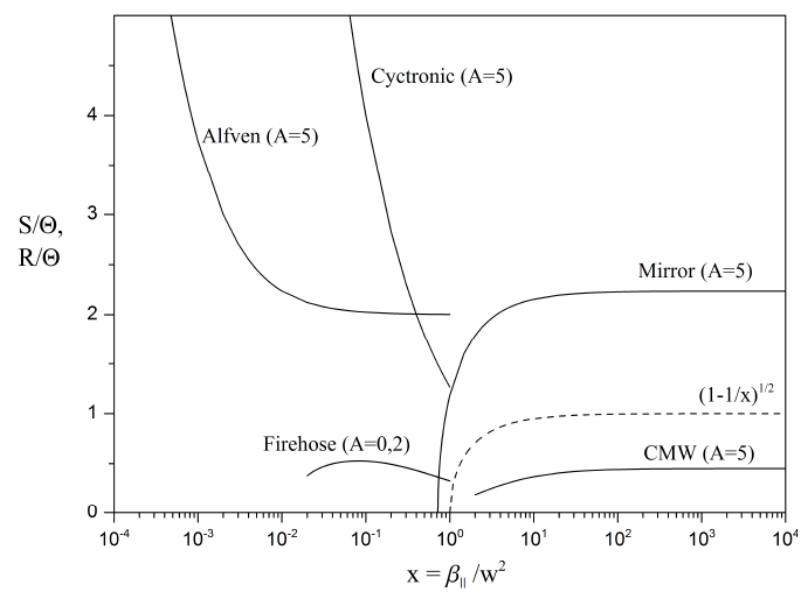

Fig. (7). Dispersion relations of the weakly amplified pair Alfven waves $R / \Theta$ and the weakly propagating mirror, firehose, CMW (cold magnetized Weibel) and cyctronic fluctuations $S / \Theta$ as a function of $x=\beta_{\|} / w^{2}$ in a bi-Maxwellian thermal pair plasma. A

value of $w=10$ and anisotropies $A=5$ for the Alfven, mirror, cyctronic and firehose fluctuations and $A=0.2$ for the CMWfluctuations are adopted. The dashed line refers to the condition $Y=1$ from Fig. (4).

\subsection{Anisotropy Diagrams}

In order to illustrate the combined restrictions on the anisotropy and plasma beta values in an anisotropic biMaxwellian magnetized pair plasma we show in Figs. $(\mathbf{8}, \mathbf{9})$ the anisotropy-plasma beta-diagram for a large $(w=10 \gg 1)$ and a small $(w=0.1 \ll 1)$ value of the electron plasma frequency phase speed $w$. Because pair-Alfven and firehose fluctuations only exist for values of $w>1$ they do not restrict the anisotropy and plasma beta values in Fig. (9).

In the case $w=10 \gg 1$ shown in Fig. (8) the stable region in the anisotropy-plasma beta parameter plane is bounded:

(a) by the pair Alfven wave instability at small plasma betas $\beta_{\|}<1$ and anisotropies $A>1$ larger than unity;

(b) by the firehose instability at large plasma betas $\beta_{\|}>1$ and anisotropies $A<1$ smaller than unity;

(c) by the mirror, CMW and pair cyctronic instability at large plasma betas $\beta_{\|}>1$ and anisotropies $A>1$ larger than unity.

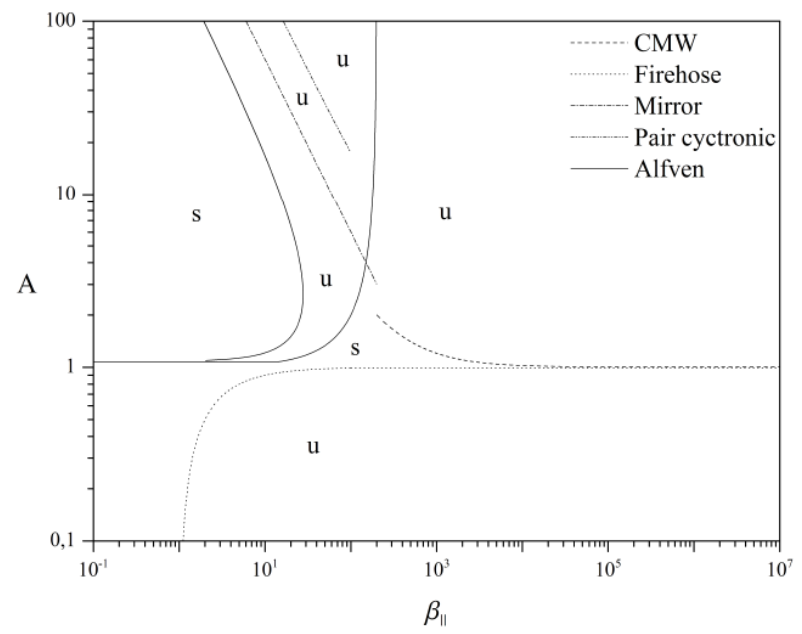

Fig. (8). Anisotropy diagram for all unstable modes in the case of a large $(w=10 \gg 1)$ value of the electron plasma frequency phase speed $w$. Stable regions are marked by "s", unstable regions are marked by "u". The bi-Maxwellian plasma is stable for values of $A$ and $\beta_{\|}$in two regions: (a) left from the left full curve where no unstable mode can be excited, and (b) above the dotted (firehose) curve, below the right full curve (pair Alfven waves), and below the dashed (cold magnetized Weibel) and dot-dashed (mirror) curves.

Apparently, there is no instability with wavevectors parallel to the background magnetic field, at small plasma betas $\beta_{\|}<1$ and small anisotropies $A<1$, i.e. $T_{\perp}>T_{\|}$. In future work, we therefore will investigate instabilities in biMaxwellian pair plasmas with wavevectors perpendicular to the background magnetic field where a possible instability has been noted before by Hamasaki [26]. 
In the case $w=0.1 \ll 1$ shown in Fig. (9) the stable region in the anisotropy-plasma beta parameter plane is only bounded at large anisotropy values $A>1$ by the mirror, CMW, pair cyclotron and pair cyctronic instabilities because pair-Alfven and firehose fluctuations do not exist for $w \ll 1$. Again a study of fluctutions with wavevectors perpendicular to the background magnetic field is needed here.

\section{SUMMARY AND CONCLUSIONS}

We rigorously studied the dispersion relations of weakly amplified and weakly propagating fluctuations with wave vectors $\vec{k} \times \vec{B}_{0}=0$ in an anisotropic bi-Maxwellian magnetized pair plasma. We extended earlier work based on the weakly-amplified limit to the case of weakly propagating $(R \ll S)$ solutions which includes in particular aperiodic fluctuations with $R=0$ by the appropriate Taylor expansion

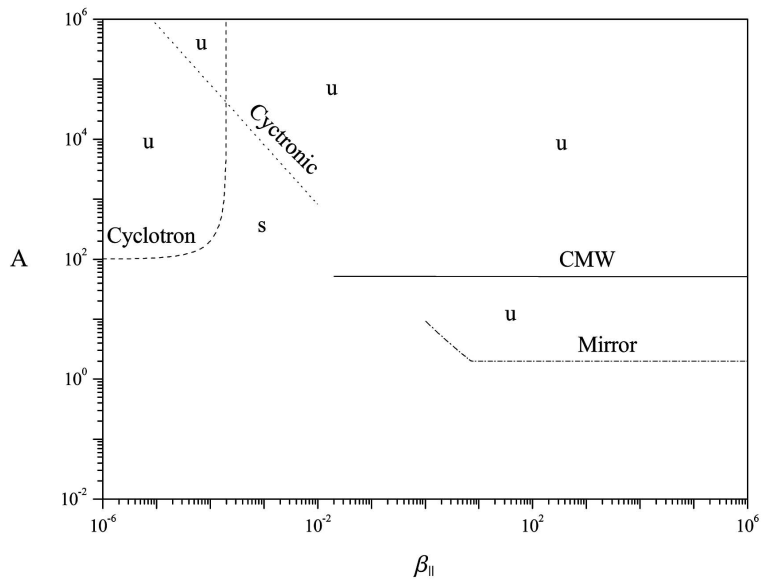

Fig. (9). Anisotropy diagram for all unstable modes in the case of a small $(w=0.1 \ll 1)$ value of the electron plasma frequency phase speed $w$. Pair-Alfven and firehose fluctuations can not be excited for $w \ll 1$ so that only the pair cyclotron, mirror, CMW and pair cyctronic instabilities contribute. Stable regions are marked by "s", unstable regions are marked by "u". The bi-Maxwellian plasma is stable for values of $A$ and $\beta_{\|}$in the region left from the dashed and dot-dashed curves which result from the instability threshold of the mirror mode.

of the plasma dispersion function. Our investigation of the resulting dispersion relation of right-handed $(\mathrm{RH})$ and lefthanded (LH) polarized fluctuations in the bi-Maxwellian pair plasma with equal parallel temperatures has demonstrated that six different modes can be excited whose properties are summarized in Tables 1-6.

The pair Alfven and cyclotron wave instabilities are the only weakly amplified solutions, whereas the four weakly propagating solutions are mirror, pair cyctronic, firehose and cool magnetized Weibel fluctuations, respectively. These four weakly propagating solutions are aperiodic with vanishing real phase speeds $R=0$ if the plasma positrons and electrons have the same temperature anisotropy $A_{p}=A_{e}=1$. The mirror and cool magnetized Weibel fluctuations reduce to the known hot and cool Weibel instabilities in the limit of an unmagnetized plasma $\left(\beta_{\|} \rightarrow \infty\right)$. For the four weakly propagating instabilities, existence and instability conditions have been derived in terms of the combined temperature anisotropy $A=\left(A_{p}+A_{e}\right) / 2$, the parallel plasma beta $\beta_{\|}$and the electron plasma frequency phase speed $w$. For the weaklyamplified pair Alfven and cyclotron waves we additionally adopted equal positron and electron anisotropies $A_{p}=A_{e}=A=A_{0}$. In agreement with Brinca's general theorem [27] on the electromagnetic stability of isotropic plasma populations none of these modes can be excited for isotropic plasma distributions ( $A=1$ ).

In the case of a large electron plasma frequeny phase speed $w=\omega_{p, e} /(k c) \gg 1$ we demonstrated that the stable region in the anisotropy-plasma beta parameter plane is bounded by the pair Alfven wave instability at small plasma betas $\beta_{\|}<1$ and anisotropies $A>1$ larger than unity, by the firehose instability at large plasma betas $\beta_{\|}>1$ and anisotropies $A<1$ smaller than unity, and by the pair cyctronic, mirror and CMW instabilities at large plasma betas $\beta_{\|}>1$ and anisotropies $A>1$ larger than unity. No instability with wavevectors parallel to the background magnetic field has been found at small plasma betas $\beta_{\|}<1$ and small anisotropies $A<1$.

In the case of a small electron plasma frequeny phase speed $w=\omega_{p, e} /(k c) \ll 1$ the stable region in the anisotropyplasma beta parameter plane is only bounded at large anisotropy values $A>1$ by the cyclotron wave, mirror, CMW and cyctronic instabilities because pair-Alfven and firehose fluctuations do not exist for $w \ll 1$.

\section{ACKNOWLEDGEMENTS}

I thank Dipl.-Phys. Tomislav Skoda for preparing the graphical illustrations and checking the calculations, and Dr. Marian Lazar for valuable discussions. I also thank the referees for their valuable comments. This work was partially supported by the Deutsche Forschungsgemeinschaft through grants Schl 201/17-1, Schl 201/19-1 and Schl 201/21-1 and the research department "Plasmas with Complex Interactions" at Ruhr-University Bochum.

\section{REFERENCES}

[1] Schlickeiser R. Cosmic ray astrophysics. heidelberg: Springer 2002.

[2] Parker EN, Tidman DA. Suprathermal particles. Phys Rev 1958; 111: 1206-11.

[3] Schlickeiser R. A viable mechanism to establish relativistic thermal particle distribution functions in cosmic sources. Astron Astrophys 1985; 143: 431-4.

[4] Yoon P. Kinetic theory of hydromagnetic turbulence. I: formal results for parallel propagation. Phys Plasmas 2007; 14: 102302-11.

[5] Beck R, Krause M. Revised equipartition and minimum energy formula for magnetic field strength estimates from radio synchrotron observations. Astron Nachr 2005; 326: 414-27.

[6] Schlickeiser R, Siewert A, Thiemann H. The diffuse radio emission from the Coma cluster. Astron Astrophys 1967; 182: 21-35.

[7] Schlickeiser R, Biermann PL, Crusius-Wätzel, A. On a nonthermal origin of steep far-infrared turnovers in radio-quiet active galactic nuclei. Astron Astrophys 1991; 247: 283-90. 
[8] Chandrasekhar S. Hydrodynamic and hydromagnetic stability. Oxford: Clarendon 1961.

[9] Bale SD, Kasper JC, Howes GG, Quataert E, Salem C, Sundkvist D. Magnetic fluctuation power near proton temperature anisotropy instability thresholds in the solar wind. Phys Rev Lett 2009; 103: 211101-4.

[10] Kasper JC, Lazarus AJ, Gary SP. Wind/SWE observations of firehose constraint on solar wind proton temperature anisotropy. Geophys Res Lett 2002; 29: 20-4.

[11] Hellinger P, Trancicek P, Kasper JC, Lazarus AJ. Solar wind proton temperature anisotropy: Linear theory and WIND/SWE observations. Geophys Res Lett 2006; 33: L09101-4.

[12] Gary SP, Karimabadi H. Linear theory of electron temperature anisotropy instabilities: Whistler, mirror, and Weibel J. Geophys Res 2006; 111: A11224-5.

[13] Gary SP, Karimabadi H. Fluctuations in electron-positron plasmas: Linear theory and implications for turbulence. Phys Plasma 2009; 161: 42104-7.

[14] Svensson R. Steady mildly relativistic thermal plasmas - Processes and properties. Mon Not R Astron Soc 1984; 209: 175-208.

[15] Jones TW, Hardee PE. Maxwellian synchrotron sources. Astrophys J 1979; 228: 268-78.

[16] Rees MJ, Cavallo G. A qualitative study of cosmic fireballs and gamma-ray bursts. Mon Not R Astron Soc 1978; 183: 359-65.

[17] Liang EPT. Emission mechanism and source distances of gammaray bursts. Nature 1982; 299: 321-3.
[18] Lamb DQ, Masters AR. X and UV radiation from accreting magnetic degenerate dwarfs. Astrophys J 1979; 234: L117-22.

[19] Gary SP. Theory of space plasma microinstabilities. Cambridge: Cambridge University Press 1993.

[20] Fried BD, Conte SD. The plasma dispersion function. New York: Academic Press 1961.

[21] Swanson DG. Plasma waves. New York: Academic 1989.

[22] Davila JM, Scott JS. The scattering of energetic particles by waves in a finite beta plasma. Astrophys J 1984; 280: 334-8.

[23] Achatz U, Dröge W, Schlickeiser R, Wibberenz G. Interplanetary transport of solar electrons and protons - Effect of dissipative processes in the magnetic field power spectrum. J Geophys Res 1993; 98: 13261-80.

[24] Weibel E. Spontaneously growing transverse waves in a plasma due to an anisotropic velocity distribution. Phys Rev Lett 1959; 2 : 83-4.

[25] Lazar M, Schlickeiser R, Poedts S. On the existence of Weibel instability in a magnetized plasma I Parallel wave propagation. Phys Plasmas 2009; 16: 12106-9.

[26] Hamasaki S. Stability of electromagnetic waves propagating perpendicular to a uniform magnetic induction. Phys Fluids 1968; 11: 1173-6.

[27] Brinca AL. On the electromagnetic stability of isotropic populations. J Geophys Res 1990; 95: 221-3. 